\title{
Adnexal Masses: Benign Ovarian Lesions and Characterization
}

\section{Benign Ovarian Masses}

\author{
Alexander Schlattau, Teresa Margarida Cunha, \\ and Rosemarie Forstner
}

\section{Contents}

1 Introduction

2 Technical Recommendations for Ovarian Lesion Characterization

3 Defining the Origin of a Pelvic Mass:

Adnexal Versus Extra-adnexal

Versus Extraperitoneal.

4 Benign Adnexal Lesions

4.1 Non-neoplastic Lesions of the

Ovaries and Adnexa

4.2 Benign Neoplastic Lesions of the Ovaries

5 Functioning Ovarian Tumors. 000

6 Ovarian Tumors in Children, Adolescents, and Young Women

7 Adnexal Masses in Pregnancy 000

References 000
A. Schlattau, M.D. $(\bowtie) \cdot$ R. Forstner

Salzburger Landeskliniken, Paracelsus Medical University,

Müllner Hauptstr. 48, Salzburg 5020, Austria e-mail: A.Schlattau@salk.at; R.Forstner@salk.at

T.M. Cunha, M.D.

Serviço de Radiologia, Instituto Português de Oncologia de Lisboa Francisco Gentil, Rua Prof. Lima Basto, 1099-023 Lisboa, Portugal e-mail: tmargarida@gmail.com

\section{Abstract}

Incidental adnexal masses are commonly identified in radiologists' daily practice. Most of them are benign ovarian lesions of no concern. However, sometimes defining the origin of a pelvic mass may be challenging, especially on ultrasound alone. Moreover, ultrasound not always allows the distinction between a benign and a malignant adnexal tumor.

Most of sonographically indeterminate adnexal masses turn out to be common benign entities that can be readily diagnosed by magnetic resonance imaging. The clinical impact of predicting the likelihood of malignancy is crucial for proper patient management.

The first part of this chapter will cover the technical magnetic resonance imaging aspects of ovarian lesions characterization as well as the imaging features that allow the radiologist to correctly define the anatomic origin of a pelvic mass. Next, the authors will go through different benign ovarian entities and through the different histologic types of benign ovarian tumors. Finally the functional ovarian tumors and the ovarian tumors in children, adolescents, young females, and pregnant women will be covered.

\section{Introduction}

Preoperative imaging plays a pivotal role in triaging management of patients with suspected adnexal masses (Forstner et al. 2016; Kaijser et al. 2014). It also aids in planning the adequate surgical 
approach. In this context, in benign ovarian lesions laparoscopy has been widely replacing open surgery. Although a definite histopathologic diagnosis is often not possible, imaging reliably assists in predicting the likelihood of malignancy which is crucial for proper patient management (Jung et al. 2002). In the assessment of adnexal masses the following parameters should be addressed by imaging: (a) defining the exact origin of the mass, (b) if the lesion is ovarian to define if it is a physiologic or neoplastic finding, and (c) when surgery is warranted for a neoplastic lesion, defining the risk of malignancy and providing a differential diagnosis (Sala and Atri 2003).

\section{Technical Recommendations for Ovarian Lesion Characterization}

MRI of the female pelvis is best performed after at least $4 \mathrm{~h}$ of fasting and with prior injection of peristaltic inhibitors to minimize artifacts due to bowel movement. The patient lies on her back in a supine position with a pelvic, torso, or cardiac coil attached around her pelvis or abdomen. Ovarian mass characterization requires anatomical coverage of the entire ovarian mass and the uterus to be able to confirm the ovarian versus uterine origin of the mass. High-resolution fast spin echo T2-weighted images are mandatory to confirm the anatomical origin and clearly identify the ovarian nature of the mass. A basic protocol includes a T2W sagittal sequence of the pelvis and pair of $\mathrm{T} 1 \mathrm{~W}$ and $\mathrm{T} 2 \mathrm{~W}$ sequences covering the adnexal mass in the same orthogonal (axial or coronal or oblique) plane with identical slice thickness (Forstner et al. 2016). Further tissue characterization takes into account signal intensity at T1- and T1FS-weighted sequences, and after intravenous injection of gadolinium.

Fig.1 A 39-year-old woman presenting with a right adnexal mass and a sonographic diagnosis of atypical endometriosis. MRI is performed for additional lesion characterization. Axial oblique T2 (a) and T1-weighted images (b), sagittal T2-weighted images (c), contrast-enhanced T1-weighted images with fat saturation (FS) (d), gross specimen photography (e). A multilocular cystic mass of the right ovary (a) with a heterogeneous content in the most anterior loculation (arrow). The corresponding T1-weighted image (b) shows no evidence of hemorrhage excluding the diagnosis of endo-
Turbo spin echo (TSE) sequences are the sequences of choice due to their excellent spatial image resolution. Imaging parameters include a field of view of 200-300 mm with a matrix of $512 \times 256$, slice thickness between 3 and $5 \mathrm{~mm}$. T2-weighted fast spin echo (FSE) sequences use a TR/TE around 4000/90 ms, whereas the TR for T1-weighted sequences is around $500 \mathrm{~ms}$ with a minimum TE. Due to time restrictions, faster T2-weighted sequences such as ultrafast half-Fourier single-shot turbo spin echo sequences (HASTE, $3 \mathrm{~s}$ per slice) or a 3D T2 WI sequences may be used. Because of its limited image resolution, the HASTE sequence can be used when the high-resolution TSE imaging is suboptimal or to provide additional imaging planes as a complement to high-resolution TSE.

DWI should be performed with a $b$ value of 800 $1000 \mathrm{~s} / \mathrm{mm}^{2}$. Diffusion restriction is found in solid aspects of both benign and malignant adnexal masses as well as in fatty and hemorrhagic masses, e.g., in endometriomas. In one study the diagnostic confidence was increased about $15 \%$ using DW additional to conventional images (ThomassinNaggara et al. 2011). When the solid component of an indeterminate adnexal mass is of low signal intensity on $\mathrm{T} 2 \mathrm{~W}$ images and the entire mass is of low signal on DWI obtained with a $b$ value of 800 $1000 \mathrm{~s} / \mathrm{mm}^{2}$ there is a very high likelihood of benignity (Thomassin-Naggara et al. 2009). DWI is thus diagnostic in the majority of common predominantly solid benign adnexal masses such as ovarian fibroma or cystadenofibroma and for the majority of pedunculated uterine leiomyomas (Forstner et al. 2016). Moreover, a T2W solid mass with low DWI signal is highly likely to be benign irrespective of its pattern or profile of contrast enhancement. For ovarian lesion characterization, intravenous contrast injection has been shown to be useful because of its ability to identify solid intracystic portions such as papillary projections, necrosis within a solid mass,

metrioma. Sagittal T2-weighted image through the heterogeneous part of the cyst shows a hypointense solid portion (arrow) with converging thin septa of the cyst, possibly suggesting normal ovarian parenchyma (c). Only minimal contrast enhancement the solid aspects and the walls of the cyst (arrowheads) is demonstrated in (d). Gross specimen photography (e) of the right ovary shows a multilocular cyst with tiny papillary projections within the wall of the posterior loculation (arrowheads) and a white fibrous portion. Histology diagnosed a serous cystadenofibroma of the ovary 

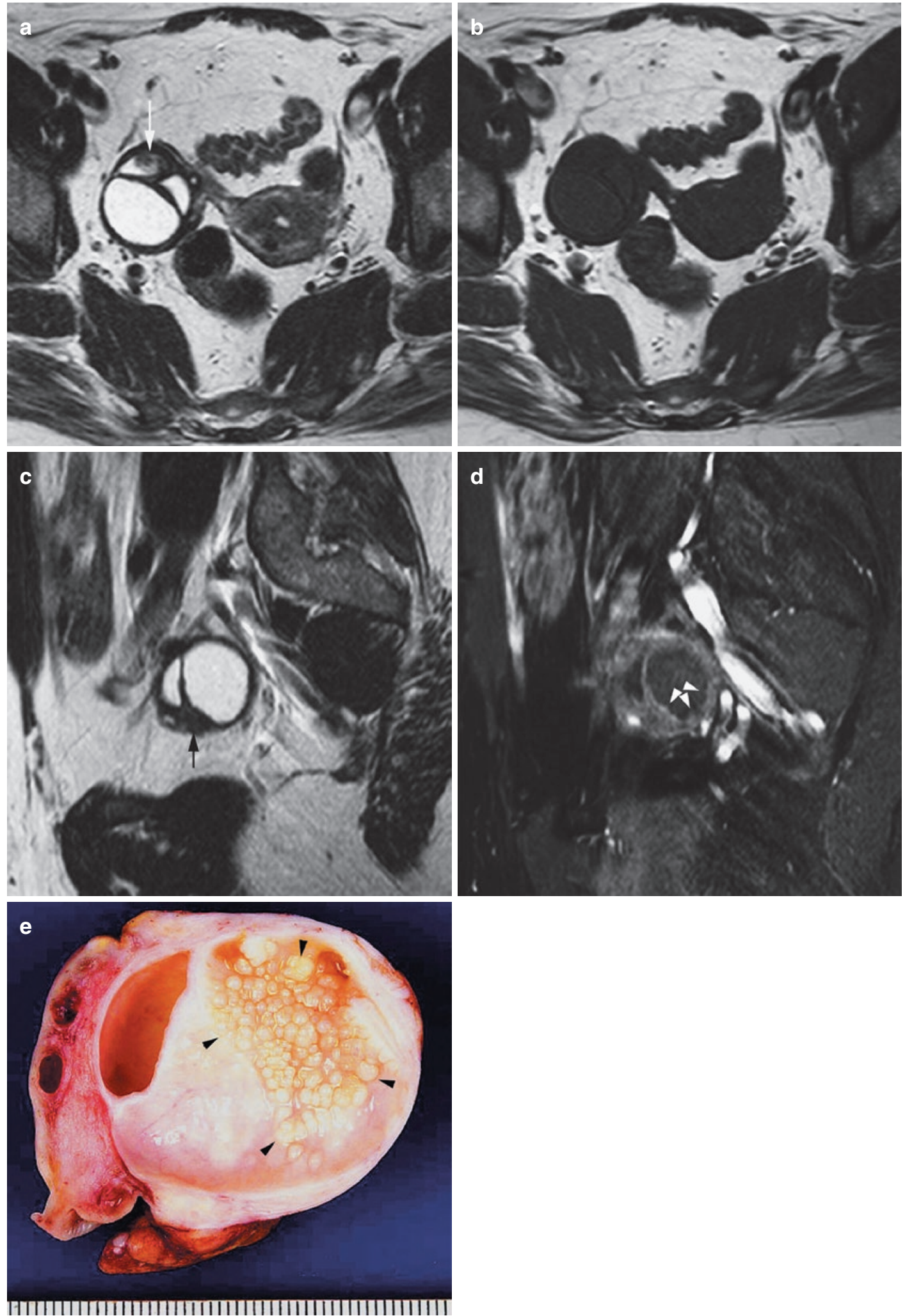
Table 1 Signal intensity of benign ovarian masses on MRI of the pelvis according to sequences and histological lesion type

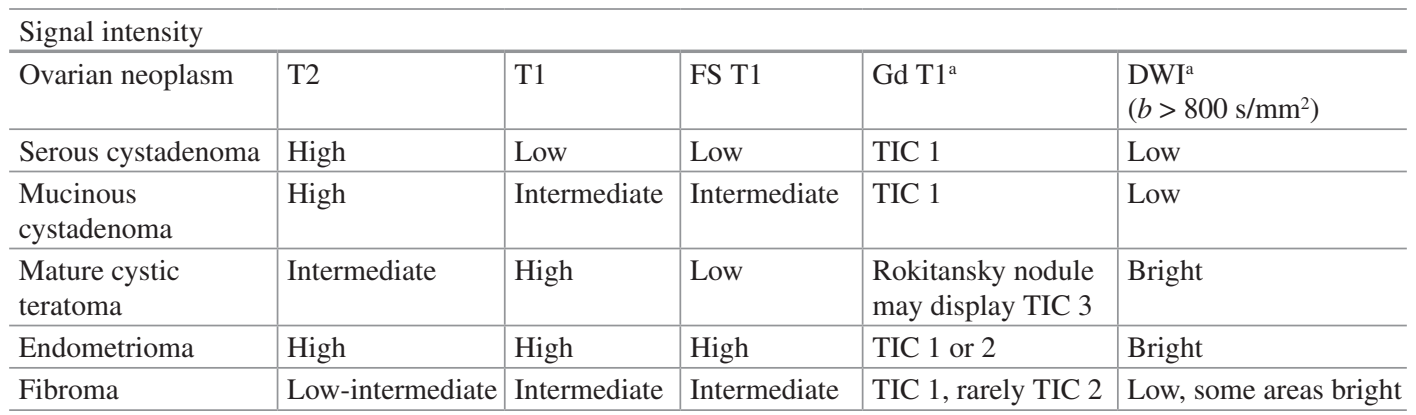

TIC time intensity curve

${ }^{a}$ Wall or septations, or solid areas in fibroma

septation, or wall thickening (Fig. 1) (Hricak et al. 2000). Recent data support that dynamic contrast enhancement is the best technique to differentiate between benign and malignant features in ovarian masses. It is based on the comparison of the time intensity curve (TIC) of the solid component in an adnexal mass with the time intensity curve of the external myometrium which is the internal reference. Thus, a DCE MR sequence should be acquired in a plane that involves the solid component of the adnexal mass (i.e., solid papillary projections, thickened irregular septa, or solid portion) and the myometrium (Forstner et al. 2016; Thomassin-Naggara et al. 2009, 2011). For details see also Chap. 66.

Dynamic contrast-enhanced imaging with acquisition of time intensity curves has been recommended by the latest ESUR guidelines on how to characterize sonographically indeterminate masses (Forstner et al. 2016). Table 1 demonstrates differences in signal intensity according to the histological type of the most frequent ovarian benign masses. Details on how to characterize adnexal masses with MRI are covered more in detail in Chap. 66.

Because of its inferior soft tissue contrast compared to MRI, CT is not the imaging modality of choice for characterization of sonographically indeterminate adnexal lesions. Indications for CT include contraindications for MRI, assessment of acute pelvic pain, of complications of pelvic inflammatory disease, and staging of ovarian cancer. Due to its wide clinical use, however, many adnexal lesions are incidentally detected by CT examinations. Published management recommendations of these incidentalomas assist in triaging these common findings (Spencer and Gore 2011; Patel et al. 2013). In CT the use of bowel opacification is generally recommended for assessment of adnexal lesions. It assists in the differentiation of fluid-filled bowel and cystic adnexal lesions and improves the identification of peritoneal seeding. Furthermore, especially in thin patients and in postmenopausal age, detection of normal ovaries is often only possible with bowel opacification. For this purpose, $1000 \mathrm{ml}$ of diluted contrast media or alternatively water is administered $1 \mathrm{~h}$ prior to the CT study. Rectal opacification is also helpful in assessing involvement of the rectum or sigmoid colon. Intravenous contrast opacification is pivotal for assessing adnexal lesions. It allows better characterization of the internal architecture and the differentiation of pelvic vascular structures, including depiction of the ovarian vascular pedicle. In most cases, a venous phase enables best depiction of the internal architecture, as solid enhancing components and papillary projections may be missed in an early phase. If a mature teratoma is suspected sonographically, a study without i.v. contrast media may be sufficient.

\section{Defining the Origin of a Pelvic Mass: Adnexal Versus Extra-adnexal Versus Extraperitoneal}

Defining the origin is the first diagnostic step in assessing a suspected adnexal mass. Depending on the site of origin the differential diagnoses and 
treatment options often differ completely. Size, architecture, and location may appear similar in adnexal, extra-adnexal peritoneal masses, and even extraperitoneal lesions. However, special features determining the anatomical relationship of the mass and the surrounding pelvic anatomical structures can assist in their differentiation (Saksouk and Johnson 2004). These parameters include visualization of ovarian structures, the type of contour deformity at the interface between the ovary and the pelvic mass, and the displacement pattern of the vessels, ureters, and other pelvic organs.

Identifying a mass separate from the ipsilateral ovary indicates its nonovarian origin (Fig. 2). However, especially in large pelvic lesions, the ovary may often be obscured or totally invaded by the mass (Levine et al. 1997). Especially in
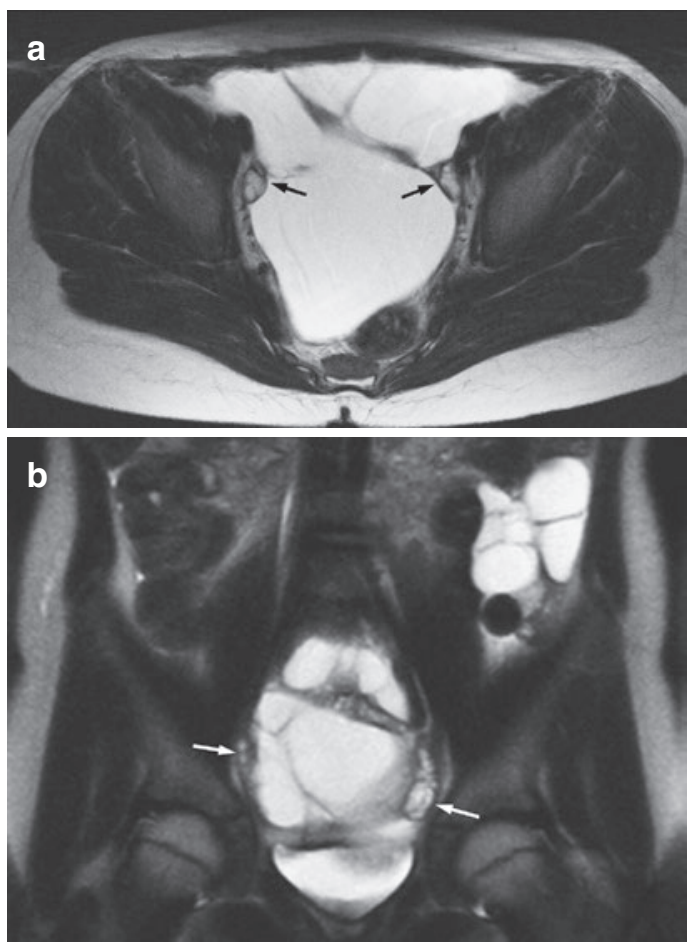

Fig. 2 Nonovarian cystic tumor. Transaxial (a) and coronal T2-weighted images (b) in a 14-year-old girl in whom sonography, to rule out an abscess after appendectomy, found a multicystic ovarian lesion. A large bilateral multiseptate lesion extending above the umbilical level is demonstrated in both planes. Identification of normal ovaries (arrows) allows exclusion of an ovarian origin of the lesion. Histopathology of the surgical specimen diagnosed a chyloma

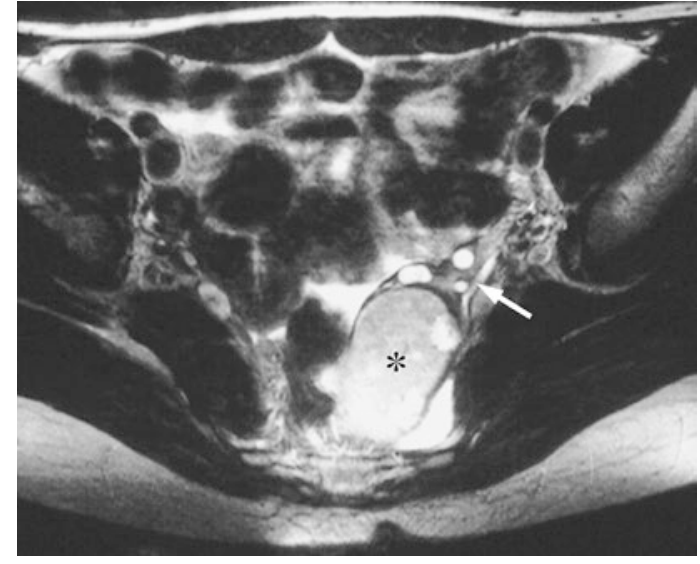

Fig.3 Beak sign. Transaxial T2-weighted images show a mass in the left cul-de-sac (asterisk). Its ovarian origin can be clearly identified due to multiple follicles (arrow). The interface to the ovarian tissue is characterized by a sharp angulation, which is typical for the beak sign. A small amount of free fluid in the cul-de-sac as seen in this patient is a physiological finding in premenopausal age and peaks in the secretory phase

smaller lesions when the ovary is not completely obscured, identifying ovarian follicles indicates its ovarian origin (Fig. 3). Furthermore, for this purpose analyzing specific signs, such as the beak sign, and the embedded organ sign can aid in better defining its relationship with the ovary. When a mass deforms the edge of the ovary into a beak shape it is likely that it arises from the ovary (Fig. 3). In contrast, dull edges at the interface with the adjacent ovary suggest that the tumor compresses the ovary but does not arise from it (Saksouk and Johnson 2004).

Large ovarian masses typically displace the ureter posteriorly or posterolaterally. The same displacement pattern can also be caused by other intraperitoneal lesions, such as the bladder, and masses arising from the uterus or bowel (Foshager et al. 1996). The iliac vessels are typically displaced laterally by an adnexal lesion. In contrast, medial displacement of the iliac vessels is typical for extraovarian masses, originating from the pelvic wall or in lymphadenopathy (Fig. 4). The origin of a mass may be further elucidated by tracking the vascular pedicle or the ovarian suspensory ligament (Saksouk and Johnson 2004). The presence of ovarian vessels leading to or emerging from an adnexal mass was identified in 
92\% of ovarian lesions in CT (Lee et al. 2003). Defining the ovarian vascular pedicle allows also the differentiation from lesions mimicking ovarian tumors, such as subserosal uterine leiomyoma. Furthermore, in the majority of leiomyomas, a vascular bridging sign at the interface between uterus and leiomyoma or the claw sign can be observed, which is not the case in ovarian lesions (Fig. 5) (Kim et al. 2000; Forstner et al. 2016).

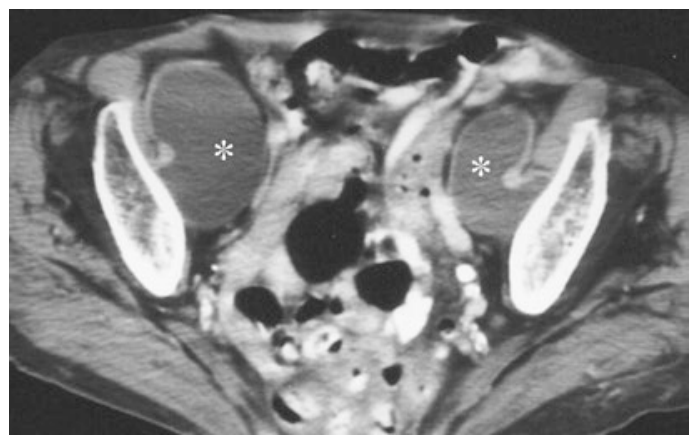

Fig. 4 Medial displacement of the iliac vessels. Transaxial CT in a patient with sonographically suspected bilateral ovarian cancer. Bilateral cystic lesions (asterisks) with mural thickening are simulating ovarian lesions. The displacement pattern of the iliac vessels, however, is typical for an origin from the pelvic sidewalls. The lesions are due to bilateral iliopectinea bursitis in a patient with rheumatoid arthritis

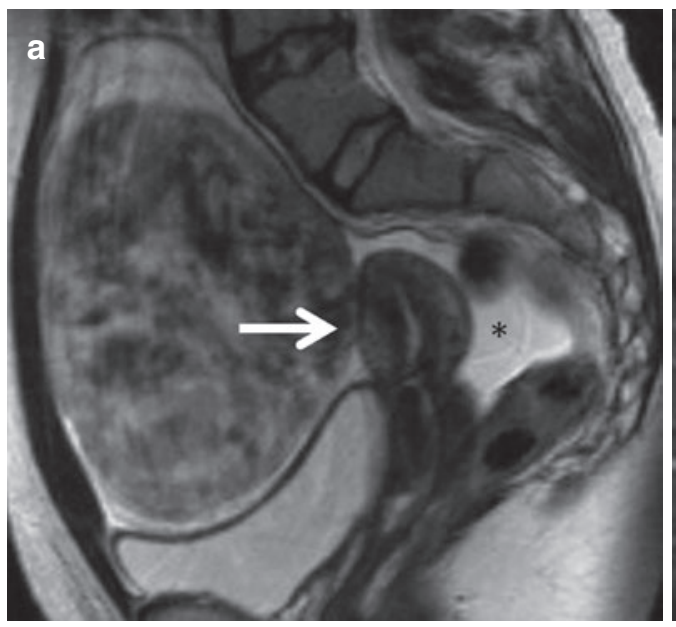

Fig. 5 Ovarian versus extraovarian mass. Sagittal T2-weighted image and axial Gd-enhanced fat-suppressed T1 image demonstrate solid lesions adjacent to the uterus in two women of reproductive age. In ovarian fibroma (a), the uterus can be separated from the ovarian mass (arrow).
Because of their close anatomic relationship, masses arising from the fallopian tubes cannot be distinguished from ovarian lesions by identifying the ovarian vessels or the ovarian suspensory ligament. Incomplete septa emerging from the wall of a cystic adnexal mass indicate the fallopian origin of the mass (Ghattamaneni et al. 2009) (Fig. 6).

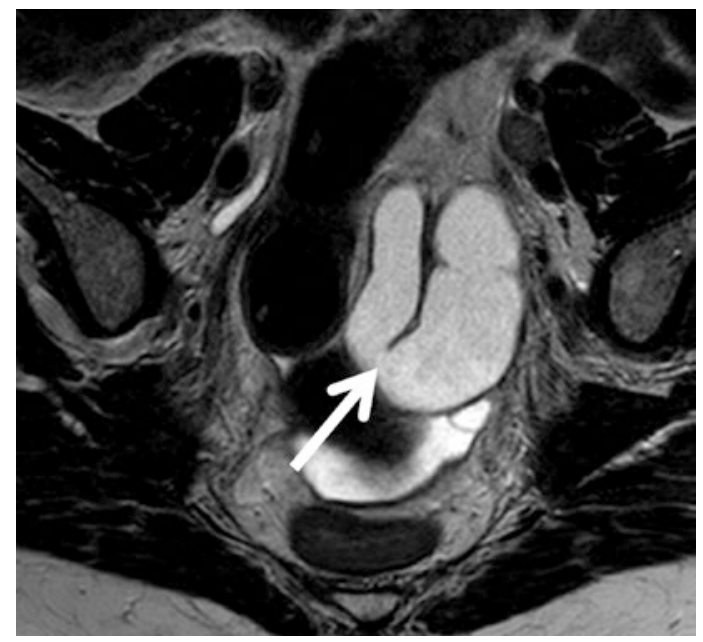

Fig. 6 Incomplete septations. Coronal oblique T2-weighted image of a left adnexal mass. Incomplete interdigitating septa (arrow) are typical findings of a hydrosalpinx

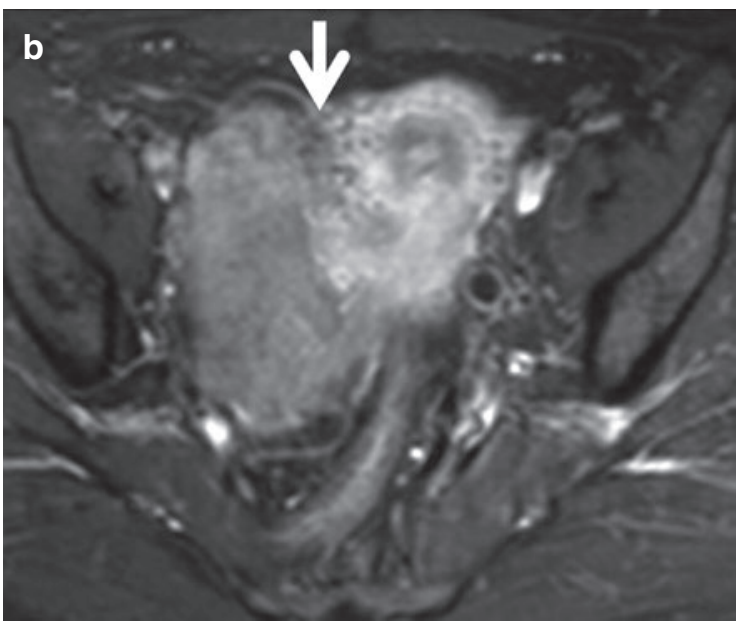

Ascites is found in the cul-de-sac and surrounding the ovarian fibroma (a). A subserosal uterine leiomyoma (b) can be differentiated from an ovarian mass by the demonstration of multiple bridging vessels. These (arrow) are found at the interface between the lesion and the myometrium 
4

\subsection{Non-neoplastic Lesions of the Ovaries and Adnexa}

\subsubsection{Physiological Ovarian Cysts: Follicular and Corpus Luteum Cysts}

The ovaries change their appearance periodically during their ovarian cycle. The ovarian cycle consists of development of the ovarian follicle, rupture, discharge of the ovum, formation, and regression of corpus luteum. Ovaries may contain follicles of various stages of development, corpus luteum cysts, and surface inclusion cysts.

Physiological ovarian cysts constitute the vast majority of cystic adnexal lesions. In the reproductive age ovarian cysts are defined as ovarian cysts if they are larger than $3 \mathrm{~cm}$ in size, whereas smaller lesions present follicles (Levine et al. 2010). Cysts may be classified as functional, which means they are associated with hormone production, or nonfunctional. In nonpregnant patients, corpus luteum cysts derive from failure of regression or hemorrhage into the corpus luteum.

Functional cysts are asymptomatic in the majority of cases. Progesterone production may persist in corpus luteum cysts, resulting in delayed menstruation or bleeding anomalies. Large physiologic cysts may cause abdominal pressure or low back pain. Acute abdomen is caused by complications such as rupture, hemorrhage, or torsion.

With an estimated prevalence of $18 \%$ ovarian cysts are a common finding in the reproductive age. Less commonly small cystic lesions may be also found in the postmenopausal age. In a series of 74 normal ovaries, the average size of the largest cyst was $1 \mathrm{~cm}$ (range, $0.2-4.7 \mathrm{~cm}$ ) (Outwater and Mitchell 1996). Functional cysts usually do not exceed $5 \mathrm{~cm}$ in size, but may occasionally grow as large as $8-10 \mathrm{~cm}$ (Fig. 7). In most cases, they are self-limiting and will regress spontaneously. In contrast to follicular cysts, corpus luteum cysts often require a period of up to 3 months to regress.
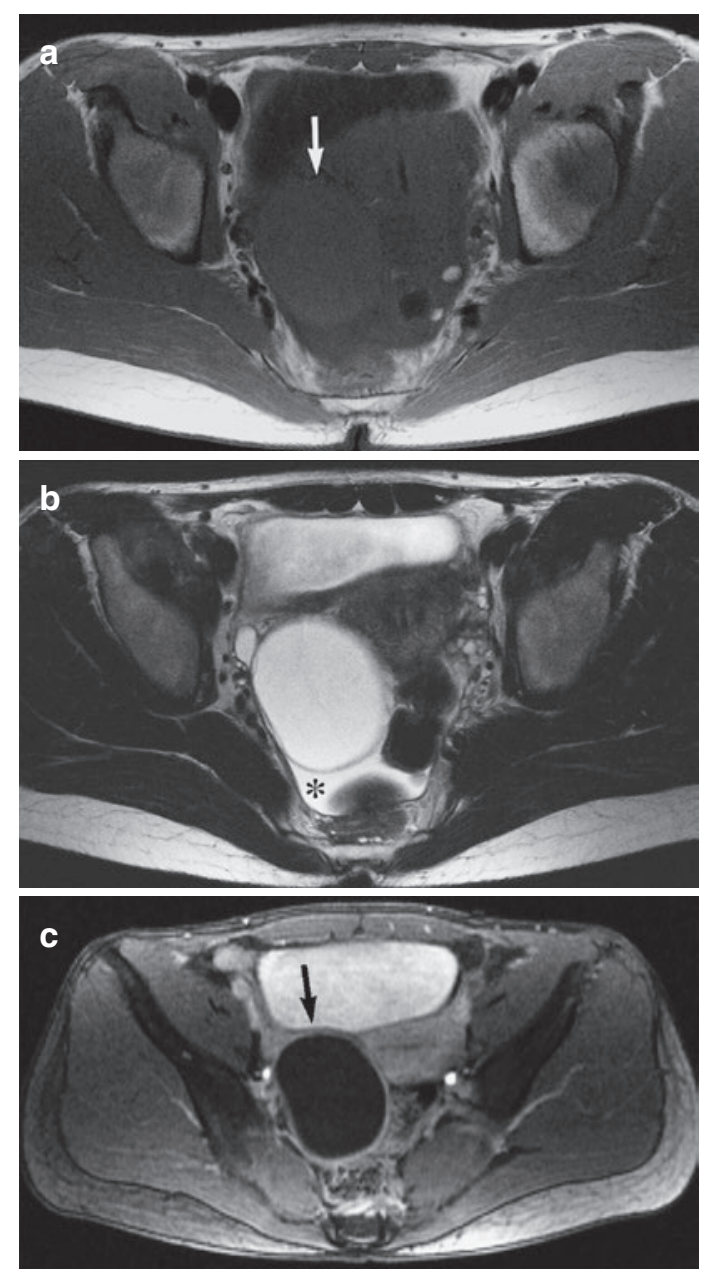

Fig. 7 Functional cyst in a 29-year-old woman. Transaxial T1 (a), T2-weighted (b), and contrastenhanced T1-weighted images with FS (c). An 8-cm cystic ovarian lesion (arrow) displays intermediate signal intensity on T1-weighted image similar to the myometrium (a) and very high SI on T2-weighted image (b). It has a thin wall well demonstrated on T2-weighted image (b) and after contrast administration (c). A functional cyst, most likely a corpus luteum cyst, could not be differentiated from an ovarian cystadenoma. The sonographic follow-up showed a considerable decrease in size within 3 months. Small amount of physiologic fluid is seen in the cul-de-sac

\subsubsection{Imaging Findings in Physiological Ovarian Cysts}

Transvaginal sonography is the gold standard for the diagnosis of ovarian cysts. According to recent management guidelines simple ovarian cysts of less than $5 \mathrm{~cm}$ in size do not require 


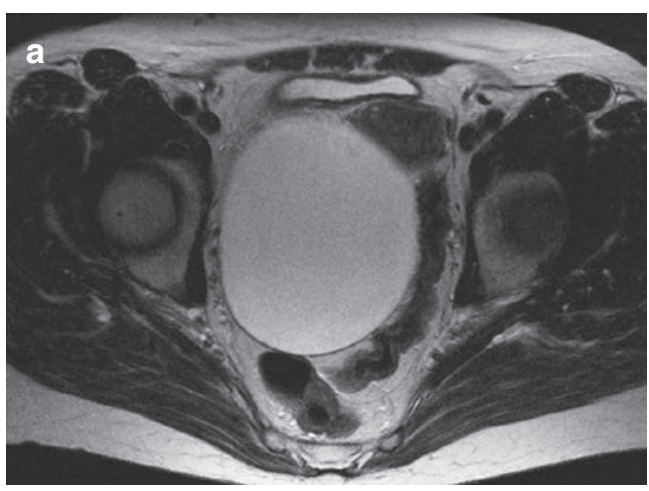

Fig. 8 Large cystic adnexal lesion in a 55-year-old woman. Transaxial T2-weighted image (a) and T1-weighted image with Gd and fat saturation (b) show a unilocular right adnexal cyst. It displaces the uterus and

further assessment in an asymptomatic woman of reproductive age (Spencer and Gore 2011; Levine et al. 2010). Findings include anechoic thinwalled cysts for simple follicular cysts and a fishnet-like heterogeneous hypoechoic content for hemorrhagic follicular or luteal cysts also described with a fine trabecular jelly-like content (Levine et al. 2010). Most of these cysts will disappear or decrease in size at short-term followup. In a follow-up $65 \%$ of the cysts persisting after menstruation had resolved within 3 months, independently of the use of oral contraceptives (Christensen et al. 2002).

Simple ovarian cysts are a common incidental finding on CT and MRI. They are unilocular and display an imperceptible or thin $(<3 \mathrm{~mm})$ wall. On CT they appear as round or oval water-density lesions ( $<20 \mathrm{HU})$. Most cysts display intermediate to low signal intensity (SI) on T1-weighted images, and very high signal intensity on T2-weighted images, due to the presence of watery fluid. The thin wall is best depicted on T2-weighted images as hypointense and on contrast-enhanced images as hyperintense to ovarian stroma (Outwater and Mitchell 1996). Corpus luteum cysts demonstrate thicker walls with distinct enhancement due to their thick luteinized cell lining. Layering by debris and internal fibrin clots in corpus luteum cysts can be differentiated from papillary projections in epithelial tumors by their lack of enhancement.

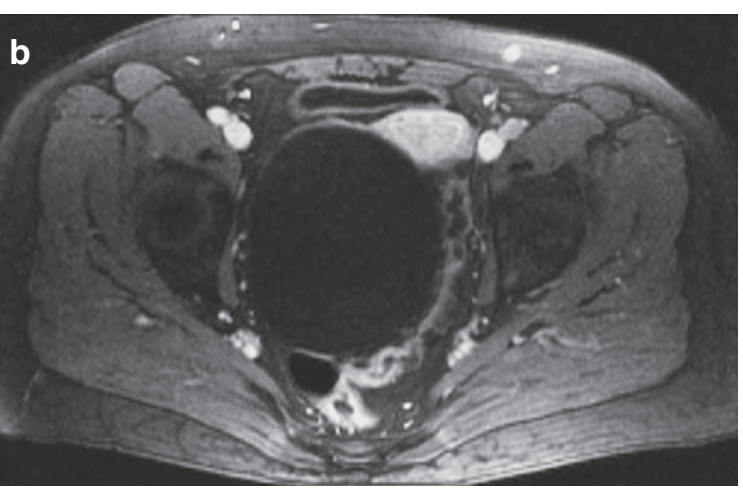

the adjacent sigmoid colon. There are no signs of malignancy. Due to postmenopausal age and the increase in size, surgery was performed. The histopathological diagnosis was serous cystadenoma

\subsubsection{Differential Diagnosis}

Unilocular cystadenomas may mimic simple ovarian cysts (Fig. 8). Regression in a follow-up over two to three cycles confirms the diagnosis of a physiological cyst.

Both corpus luteum cysts and endometrioma may show intracystic hemorrhage. However, endometrioma tend to be bilateral, are often multiple, and typically demonstrate prominent $\mathrm{T} 2$ shortening ("shading") and the T2 dark spot sign presenting mural clot (Corwin et al. 2014).

\subsubsection{Paraovarian Cysts}

Paraovarian cysts (paratubal) cysts arising from Wolffian duct remnants in the mesovarium (Moyle et al.2010) are common incidentalomas. Although encountered throughout life, they are most commonly found in middle-aged women. Surgical data suggest that they account for 10-20\% of adnexal masses (Kier 1992). They are round or ovoid, unilocular thin-walled cysts ranging between 1 and $12 \mathrm{~cm}$ in size (Kim et al. 1997). Complications do not differ from those of other ovarian cysts. Secondary transformation with foci of benign and malignant papillary neoplasms is extremely rare (Honoré and O’Hara 1980).

\subsubsection{Imaging Findings}

Paraovarian cysts tend to be large thin-walled unilocular cysts, located typically within the broad ligament (Fig. 9). Rarely they may contain 


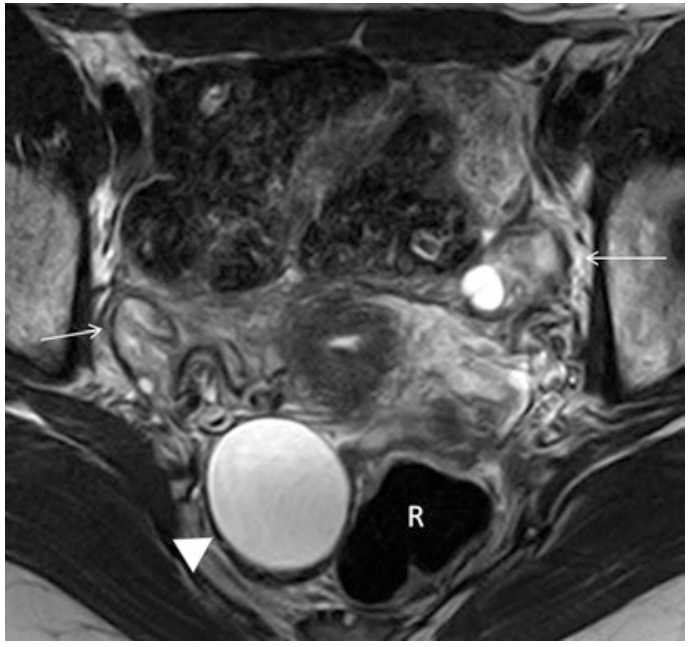

Fig. 9 Paraovarian cyst in a 53-year-old female. Transaxial T2WI demonstrates a $5 \mathrm{~cm}$ cyst (arrowhead) that is clearly separated from the postmenopausal right ovary (arrow). A $1 \mathrm{~cm}$ cyst with a thin septum is seen on the left ovary (arrow). Rectum $(R)$

internal septations. On CT and MRI, they display typical criteria of ovarian cysts, but are found separate from the ipsilateral ovary (Moyle et al. 2010).

\subsubsection{Differential Diagnosis}

A paraovarian cyst can only be distinguished from an ovarian cyst if it is clearly separated from the ovary. While paraovarian cysts are usually larger cysts, cysts of Morgagni, which arise from the fimbriated end of the tube, usually do not exceed $1 \mathrm{~cm}$ in diameter. The differential diagnosis of paraovarian cysts includes ovarian cystadenoma, an eccentric ovarian cyst, retroperitoneal cysts, and lymphoceles. The latter can be differentiated based on the clinical history and the pattern of vascular displacement. Hydrosalpinx may have a similar location within the broad ligament; however, it displays a tubular form and interdigitating septa (Moyle et al. 2010). In contrast to paraovarian cysts, the shape of peritoneal inclusion is defined by the surrounding structures.

\subsubsection{Peritoneal Inclusion Cysts}

Peritoneal inclusion cysts (pseudocysts or multilocular inclusion cysts) are accumulations of fluid produced by the ovaries that become entrapped by peritoneal adhesions. These lesions are of variable size and are typically encountered in premenopausal women with previous surgeries, endometriosis, or pelvic inflammatory disease (PID) (Moyle et al. 2010). Pseudocysts have an irregular shape because the outer surface is not a true wall but defined by surrounding structures. They may become clinically apparent due to mass effect, chronic pelvic pain, or present without symptoms (Kim et al. 1997).

\subsubsection{Imaging Findings}

Peritoneal inclusion cysts tend to take the shape of the space they are occupying. The ovary is entrapped and lies typically inside the cyst or within the cyst wall and may be mistaken as a solid nodule (Fig. 10). The internal architecture of peritoneal inclusion cysts depends on its contents. In most cases, they contain simple fluid with low SI on T1-weighted images and very high SI on T2-weighted images, and low density on CT. Hemorrhage and layering of hemosiderin can lead to high SI on T1 and low SI on T2 W, and higher densities on CT. In one study internal septa were found in 11/15 cases of peritoneal inclusion cysts (Kim et al. 1997).

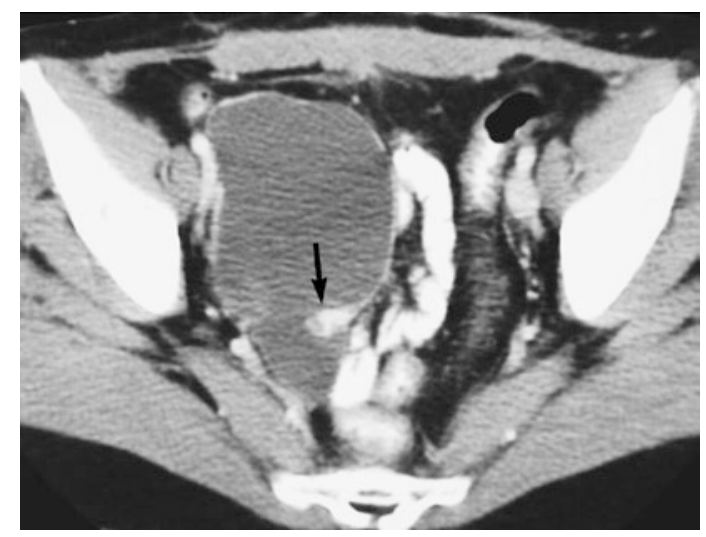

Fig. 10 Peritoneal inclusion cyst on CT. A cystic lesion of the right adnexa was found on sonography in a 33-year-old woman with a history of several previous pelvic surgeries. CT demonstrates a cystic lesion with thin enhancing walls and a solid ovoid structure (arrow) at its posterior wall. Surgery revealed a peritoneal inclusion cyst. The solid structure was the normal ovary 


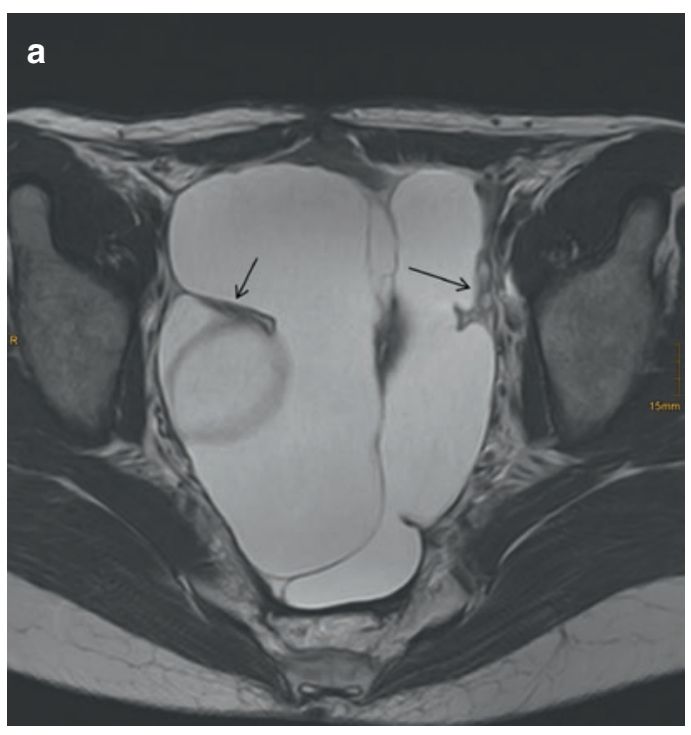

Fig. 11 Peritoneal pseudocyst on MRI. Transaxial (a) and coronal T2WI (b) show a multiseptate cystic bilateral mass. Imaging features typical of this entity include the

\subsubsection{Differential Diagnosis}

Particularly in CT differentiation of a pseudocyst from cystadenomas or ovarian cancer may be difficult due to internal septations and the murally located ovary mimicking a solid component within a cystic mass. Conforming to the peritoneal cavity and encasement rather than displacement of adjacent organs as well as a history of previous surgeries, PID, or Crohn's disease are key elements for the correct diagnosis (Fig. 11).

\subsubsection{Theca Lutein Cysts}

Theca lutein cysts are ovarian cysts that are lined by luteinized theca cells. They develop in patients with high levels of serum human chorionic gonadotropin and are associated with multiple gestations, trophoblastic disease, and pregnancies complicated by hydrops fetalis, or in patients with ovarian hyperstimulation syndrome (Fig. 12).

\subsubsection{Imaging Findings}

Theca lutein cysts are typically large, bilateral multiseptate ovarian cysts composed of simple fluid. They may cause gross enlargement of the ovaries to $10-20 \mathrm{~cm}$ in diameter. T2-weighted images or contrast-enhanced

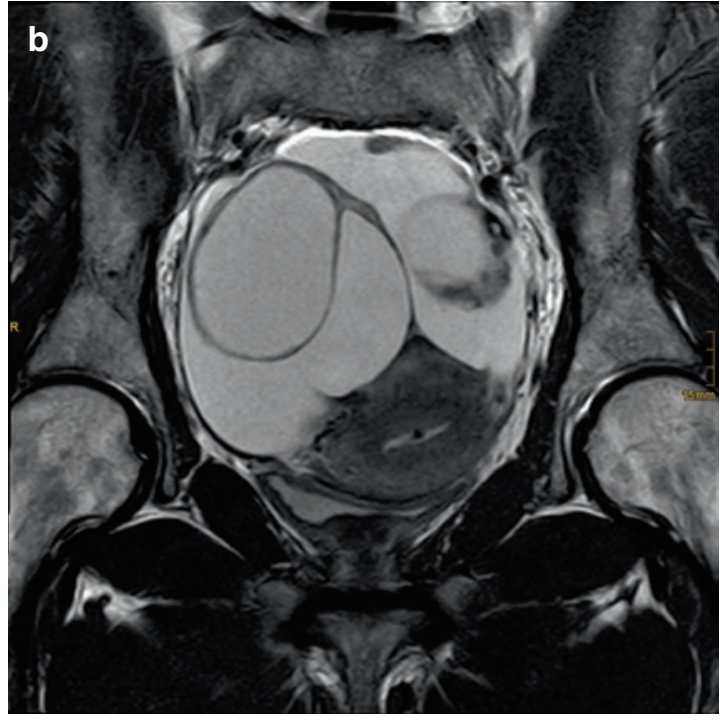

ovary (arrows) displayed at the wall or within a septum, thin septas, and the contour following the predefined anatomical space

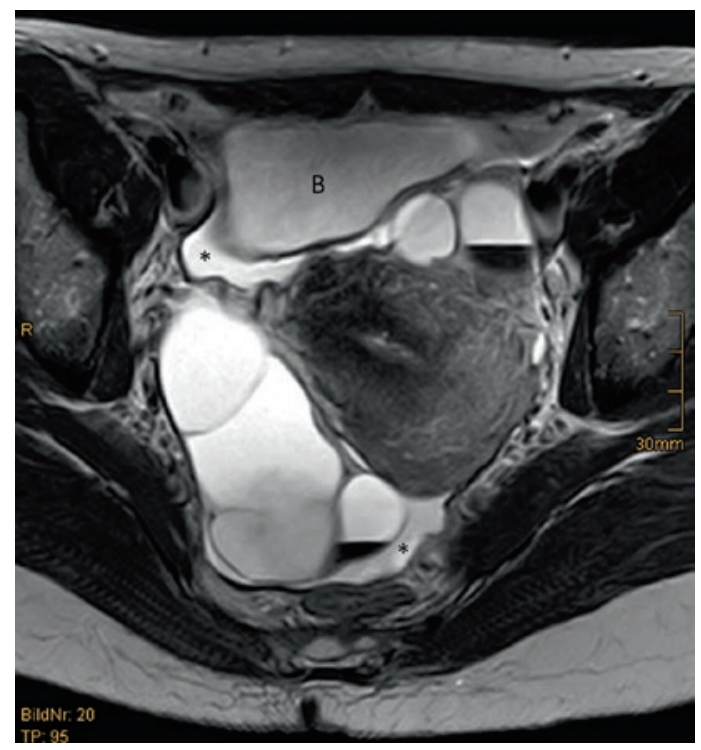

Fig. 12 Ovarian hyperstimulation syndrome in a 45-yearold female treated with tamoxifen. Bilateral enlarged ovaries with multiple thin-walled cysts are found. Some of these show hemorrhagic foci. Bladder (B). Ascites (asterisk)

MRI or CT will typically display no evidence of mural thickening (Fig. 13).

\subsubsection{Differential Diagnosis}

Theca lutein cysts may resemble bilateral cystadenomas, but the clinical background is different. 


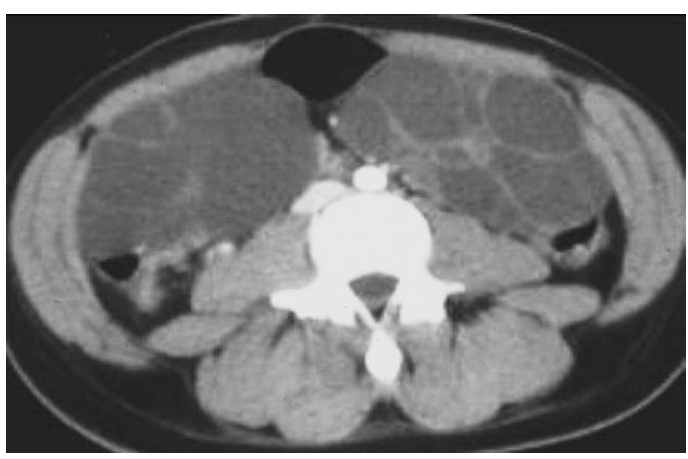

Fig. 13 Bilateral theca lutein cysts CT at the umbilical level in a 27 -year-old patient with a hydatidiform mole. Bilaterally enlarged ovaries are demonstrated displaying numerous thin-walled cysts of water-like density. No enhancing solid structures or papillary projections could be identified. Theca lutein cysts are found in up to $20 \%$ of patients with a hydatidiform mole

\subsubsection{Polycystic Ovary Syndrome}

Polycystic ovary syndrome (PCOS) is a complex endocrinologic disorder characterized by inappropriate gonadotropin secretion that results in chronic anovulation. It affects $5-10 \%$ of women of reproductive age and is found in 50\% of women with infertility problems (Lakhani et al. 2002).

Although most notable in Stein-Leventhal syndrome, which comprises the classical findings of amenorrhea, hirsutism, obesity, and sclerotic ovaries, a wide range of clinical presentations exist. Only one-quarter to one-half of the patients present the classical signs. Another feature is metabolic disorders including increased risk of diabetes, cardiovascular disease, and endometrial hyperplasia or endometrial cancer (Lee and Rausch 2012). The morphologic hallmark is mild enlargement of both ovaries, which contain numerous multiple small follicles surrounding the increased central ovarian stroma.

\subsubsection{Imaging Findings}

As there is an overlap of normal and polycystic ovaries in imaging, the diagnosis of polycystic ovary syndrome is based on hormonal changes as well as clinical and imaging findings (Johnstone et al. 2010).

The imaging modality of choice to assess PCOS is transvaginal US. The Rotterdam consensus defined a threshold of at least 12 or more follicles, measuring between 2 and $9 \mathrm{~mm}$
(FNPO), and/or an ovarian volume $>10 \mathrm{~cm}^{3}$ (Franks 2006). Newer data suggest follicle counting by $3 \mathrm{D}$ techniques and increasing the number of follicles (Lujan et al. 2013). MRI is usually not warranted to diagnose PCOS (Lee and Rausch 2012). In this setting the role of MRI is as an adjunct to US to exclude a virilizing ovarian tumor and to assess the adrenal glands.

The MRI findings in PCOS include bilateral moderately enlarged (up to $5 \mathrm{~cm}$ ) spherical ovaries with an abnormally high number of follicles measuring less than $10 \mathrm{~mm}$ in size (Fig. 14). At least 12 follicles are found in a peripheral distribution (Fig. 9.24) in each ovary. Rarely, a normal contralateral ovary may be identified. The ovaries are surrounded by a thickened sclerotic capsule and typically display abundant low signal central stroma on T1- and T2-weighted images (Fig. 14).
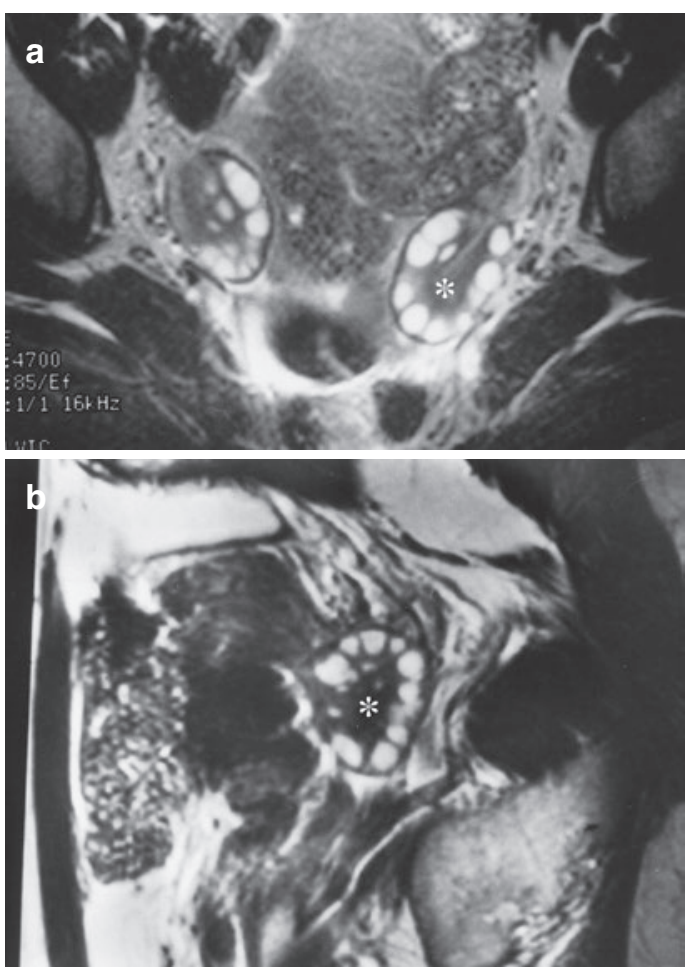

Fig. 14 Polycystic ovaries in MRI. Transaxial T2-weighted image (a) and parasagittal T2 WI (b) in a patient with Stein-Leventhal syndrome. Bilateral spherical ovaries are demonstrated showing numerous small follicles of uniform size. The latter are located in the periphery of the ovary much like a "string of pearls" surrounding the ovarian stroma (asterisk), which typically is of very low signal intensity on T2-weighted images in PCO 


\subsubsection{Differential Diagnosis}

Multiple follicles in different stages of development including complex cysts constitute a common finding in adolescents, and present a normal finding in mid- to late puberty (Laufer 2017). Using the threshold of 12 follicles an overlap to PCOS exists also in normal young females (Duijkers and Klipping 2010). Multifollicular ovaries may also be found in hyperprolactinemia, hypothalamic anovulation, and weight-related amenorrhea. They may be differentiated from PCO by fewer cysts, the different size of follicles, lack of stromal hypertrophy, and the distribution of the often larger follicles throughout the ovary (Clement 2002). In contrast to PCO, the ovaries resume normal appearance after treatment.

\subsection{Benign Neoplastic Lesions of the Ovaries}

Benign ovarian neoplasm accounts for $57 \%$ of epithelial ovarian tumors and for $80 \%$ of all ovarian tumors (Seidman et al. 2002). Although there is large spectrum of benign ovarian neoplasm, the vast majority are encompassed by only a few different histologic types. It is a matter of debate whether cystadenomas or teratomas are most frequent. In a large series cystic teratomas accounted for the majority of benign tumors (58\%) followed by serous cystadenomas $(25 \%)$ and mucinous cystadenomas (12\%), benign stromal tumors (fibromas/thecomas) (4\%), and Brenner tumors (1\%) (Koonings et al. 1989). In epithelial tumors a continuum between various subtypes of benign cystadenomas, borderline, and malignant epithelial tumors is found (Lengyel 2010).

\subsubsection{Cystadenoma}

Cystadenomas account for 37-50\% of benign ovarian tumors in the reproductive age. Their frequency tends to increase with age, and after menopause, cystadenomas account for up to $80 \%$ of benign ovarian tumors (Jung et al. 2002). Cystadenomas are thin-walled unilocular or multilocular cystic lesions filled with serous, mucinous, and sometimes hemorrhagic contents. Small papillary projections within the cyst walls may be rarely found in serous cystadenomas (Hassen et al. 2011). Serous and mucinous cystadenomas differ in pathology, prognosis, and disease course. Serous cystadenomas are more common than mucinous cystadenomas, with a prevalence of $11.2 \%$ vs. $7.4 \%$ in the IOTA series analyzing more than 3000 patients with adnexal masses (Timmerman et al. 2016). Both types of cystadenomas have been recognized as precursors of ovarian cancer and may slowly transform to borderline tumors and invasive ovarian cancer (Lengyel 2010).

Serous cystadenomas account for up to $40 \%$ of all benign ovarian neoplasms. They show a peak incidence in the fourth and fifth decades and are in up to $20 \%$ bilateral. Mucinous cystadenomas account for $20-25 \%$ of all benign ovarian neoplasms, and are bilateral in only $5 \%$ of cases (Hochberg and Hoffman 2017). Both are cystic lesions filled with water-like or higher proteinaceous contents. Small coarse calcifications in mural location or within the cyst were found in $>30 \%$ of mucinous cystadenomas, whereas tiny psammoma bodies in serous cystadenomas are a rare finding in CT (Okada et al. 2005). Mucinous cystadenomas tend to be larger at presentation and are typically multilocular with different contents of the loculi (Fig. 15) (Jung et al. 2002). These loculi are small and multiple and separated by thin septations. Rupture of a mucinous cystadenoma can result in pseudomyxoma peritonei.

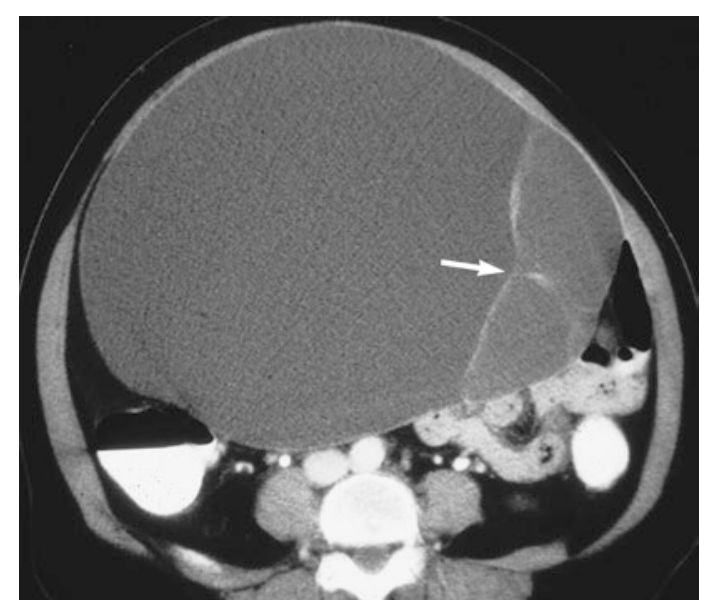

Fig. 15 Mucinous cystadenoma on CT. At the level of L5, a cystic ovarian lesion extending to the upper abdomen and measuring $25 \mathrm{~cm}$ in diameter is demonstrated. It bulges the abdominal wall and displaces bowel loops posteriorly. It displays multiple thin septations (arrow). Loculi in the left periphery display attenuation values which are higher than water. The large lesion size and different densities of the loculi are findings suggesting the diagnosis of a mucinous cystadenoma 


\subsubsection{Imaging Findings}

Although an overlap exists, imaging features aid in the differentiation of serous from mucinous cystadenomas (Jung et al. 2002). In general, serous cystadenomas follow the pattern of a simple cyst and mucinous cystadenomas those of a multilocular lesion. For details see Chap. 66. Cystadenomas are well-circumscribed cystic
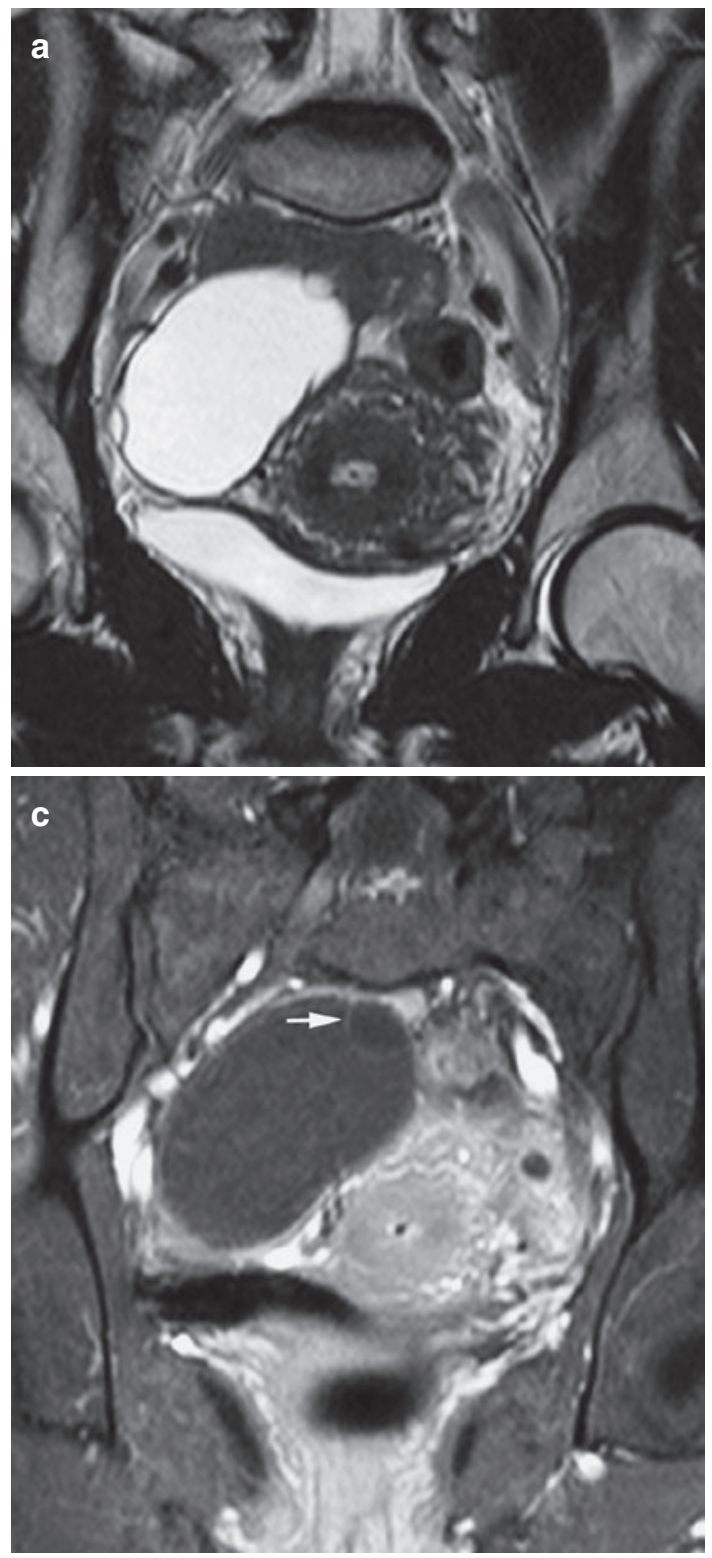

Fig. 16 Mucinous cystadenoma. In a 48-year-old woman with a cystic mass in sonography, complementary MRI with coronal T2-weighted image (a), coronal T1-weighted image (b), and fat-suppressed contrast-enhanced T1-weighted image (c) are demonstrated. Coronal T2-weighted MR image of the pelvis shows a hyperin- tumors with enhancing thin walls and-if present-internal septations on CT and MRI (Fig. 15). The wall and septa are regular and thin $(<3 \mathrm{~mm})$ (Fig. 16). Papillary projections may be found in cystadenomas, but these tend to be small and a size of less than 3-5 mm indicates benignity (Timmerman et al. 2016) (Figs. 17 and 18).

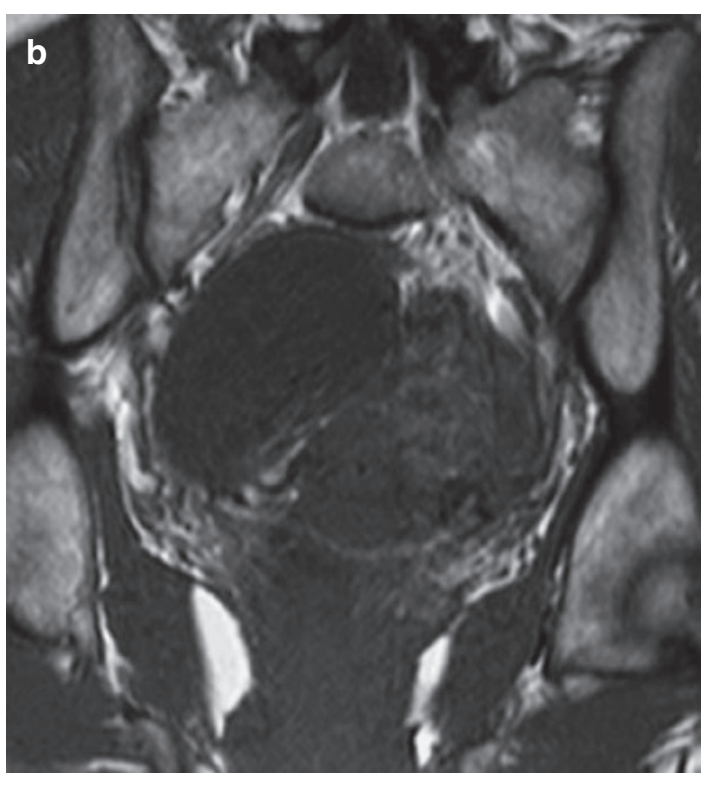

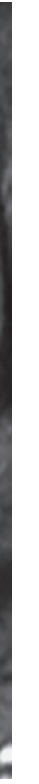

tense multilocular cystic mass with thin septation (a) in the right adnexal region. T1-weighted image confirms the purely cystic content of the cyst (b). The septations are thin and demonstrate contrast enhancement (c). Pathology after laparoscopic removal of the ovary showed benign mucinous cystadenoma of the ovary 


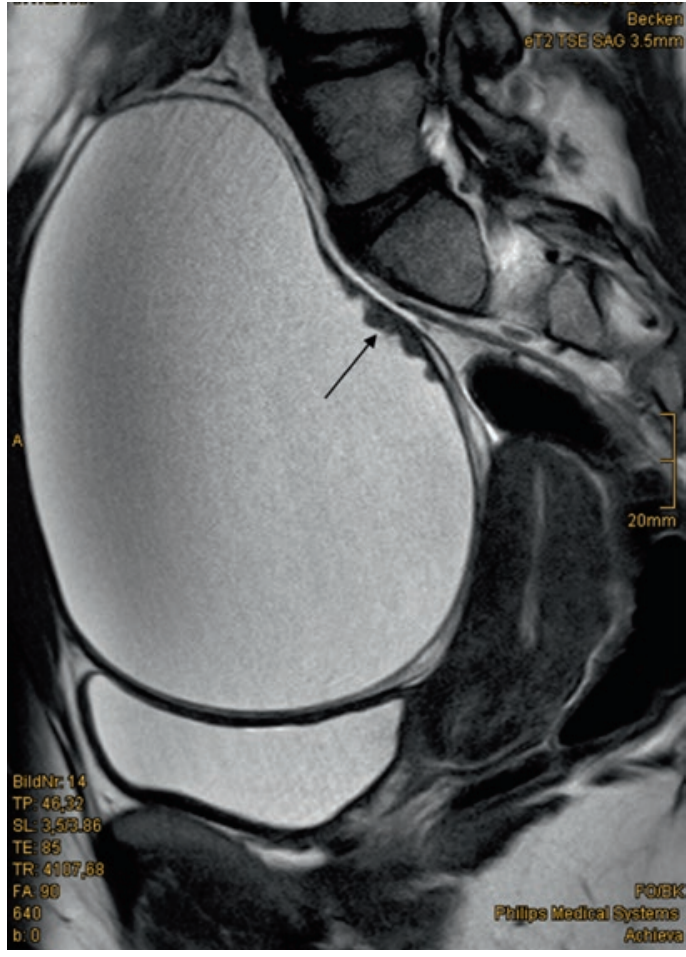

Fig. 17 Papillary projections in a mucinous cystadenoma. MRI was performed complementary to sonography in a persistent ovarian cyst. Sagittal T2WI displays a large unilocular cyst that shows small irregular mural thickening of the posterior wall presenting papillary projections (arrow)

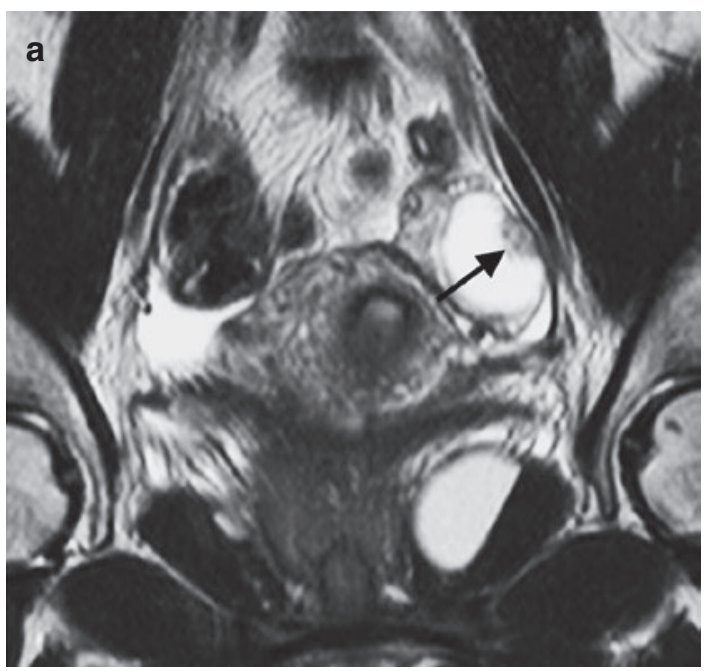

Fig. 18 Vegetation within a cyst. Coronal T2-weighted image (a) and fat-saturated contrast-enhanced T1-weighted image (b). In a $3.5 \mathrm{~cm}$ ovarian cyst, a mural nodule of $10 \mathrm{~mm}$ with intermediate signal intensity on T2-weighted
The cystic loculi of serous cystadenomas display signal of simple fluid. In contrast, mucinous cystadenomas have often various signal intensities depending on the contents within the different loculi, which vary from watery to proteinaceous to hemorrhagic. They display SI intensity higher than water on $\mathrm{T} 2$ and lower SI on T2-weighted images relative to serous fluid. When hemorrhage is present, blood products may be identified on MRI. Rarely, mucinous cystadenomas can manifest as a simple cyst.

Mucinous cystadenomas tend to be large at diagnosis with a mean size of $10 \mathrm{~cm}$, but may be as large as $30 \mathrm{~cm}$ (Jung et al. 2002).

\subsubsection{Differential Diagnosis}

Serous and mucinous cystadenomas may display similar imaging findings on CT and MRI. If papillary projections are found in cystadenomas, they tend to be fewer and smaller than in borderline tumors and display a type 1 time intensity curve. The presence of a mural nodule is highly indicative of a borderline or invasive malignancy (Fig. 18). Endometriomas may resemble mucinous cystadenomas, especially when these are complicated by hemorrhage. Low SI shading on the T2-weighted images is

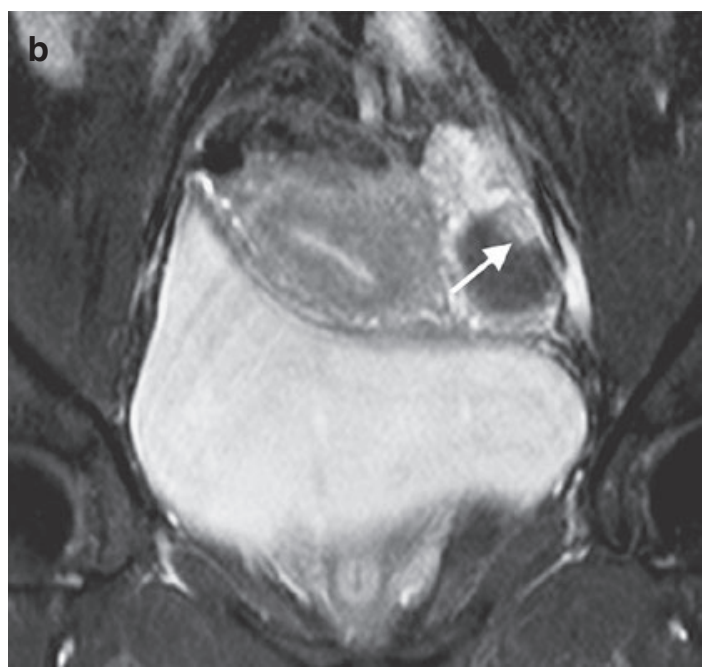

image (a) and contrast enhancement (b) is demonstrated. This finding should warrant the suspicion of malignancy, especially a borderline tumor as in this patient. Papillary projections in cystadenomas tend to be smaller 
not exclusive of endometriomas. Homogenous shading is the most prevalent pattern in endometriomas but focal/multifocal shading within a complex mass may also be found in endometroid carcinomas (Dias et al. 2015). Furthermore, the walls in endometriomas tend to be thicker and irregular and endometrioma usually are smaller than $10 \mathrm{~cm}$. In CT the differentiation of cystadenomas from endometriomas is not possible. Hydrosalpinx can also display as a multiloculated uni- or bilateral adnexal lesion. In contrast to cystadenomas, the loculi communicate and incomplete septa are found (Ghattamaneni et al. 2009).

\subsubsection{Cystadenofibroma}

Cystadenofibromas account for $1.7 \%$ of ovarian tumors. They are benign cystic tumors composed of epithelial and various amounts of solid stromal elements. They can also be purely cystic with small foci of stroma detected microscopically. The margin tends to be well defined and smooth. Endocrine activity is not found (Jung et al. 2006).

\subsubsection{Imaging Features}

Cystadenofibroma present as uni- or bilateral ovarian predominantly cystic tumors. Solid stromal tissue displaying very low SI on T2WI is the key feature suggesting cystadenofibroma. This may be encountered in a diffuse or partially thickened cyst wall or in a multicystic or solid element within the lesion (Fig. 19). The reference most suitable for low SI in a benign lesion is the skeletal pelvic muscle (Forstner et al. 2016). On MRI small papillary projections may be displayed (Fig. 1). On CT coarse calcifications suggest benignity. Variable amounts of fibrous stroma in ovarian cystadenofibromas result in imaging features that vary from purely cystic to a complex cystic tumor with one or more solid components. In one series of 32 ovarian cystadenofibromas, $50 \%$ displayed as multiloculated masses identical to cystadenomas. The other half was complex cystic tumors with one or more solid components and smooth thickened septa of very low SI. This feature has been described as black sponge sign (Cho et al. 2004).

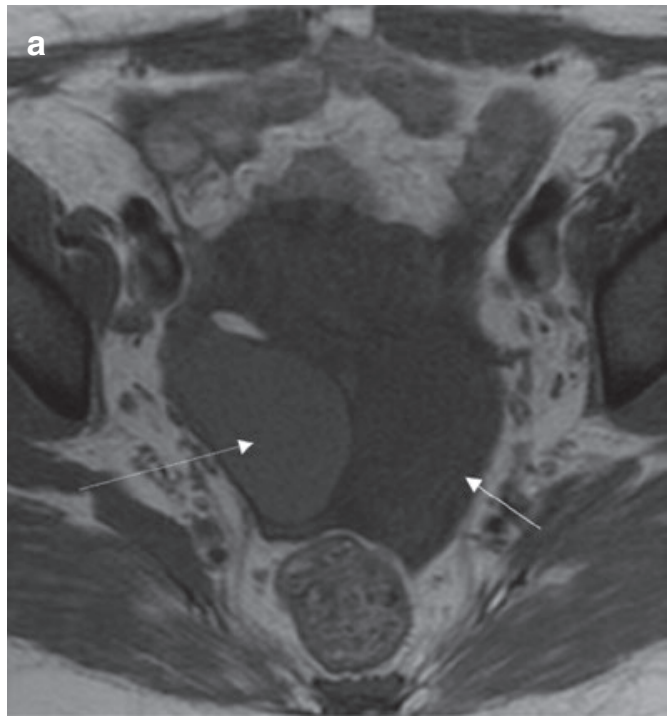

Fig. 19 A 45-year-old patient with a right cystadenofibroma and a left fibroma. (a) Axial T1-weighted image; (b) axial T2-weighted image; (c) fat-saturated axial gadolinium-enhanced T1-weighted image; (d) axial oblique T2-weighted image. T2-weighted images show a solid hypointense lesion in the left ovary that shows mod-

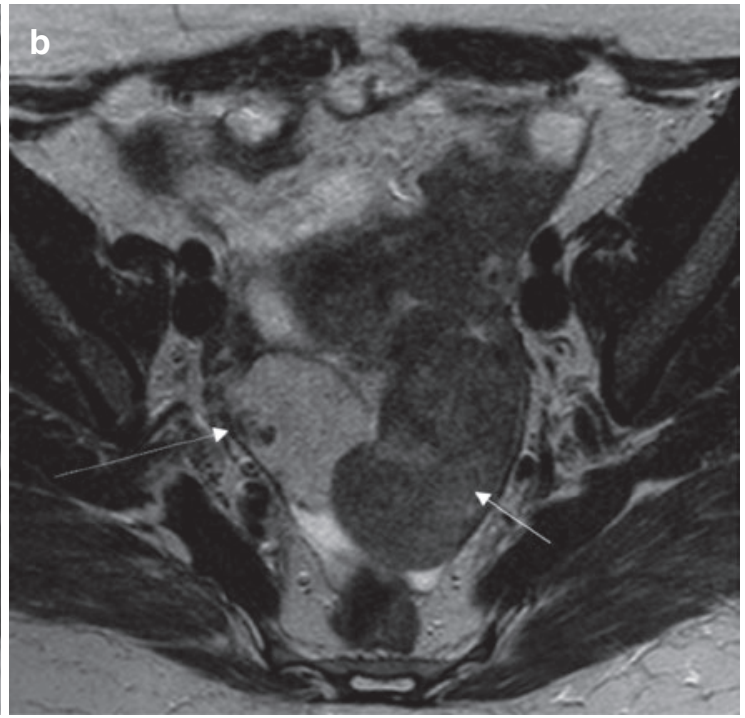

erate contrast enhancement and therefore is compatible with a fibroma (arrows). In the right ovary there is a cystic lesion with small areas of low signal intensity on T2-weighted images that revealed to be a cystadenofibroma (dashed arrows) 

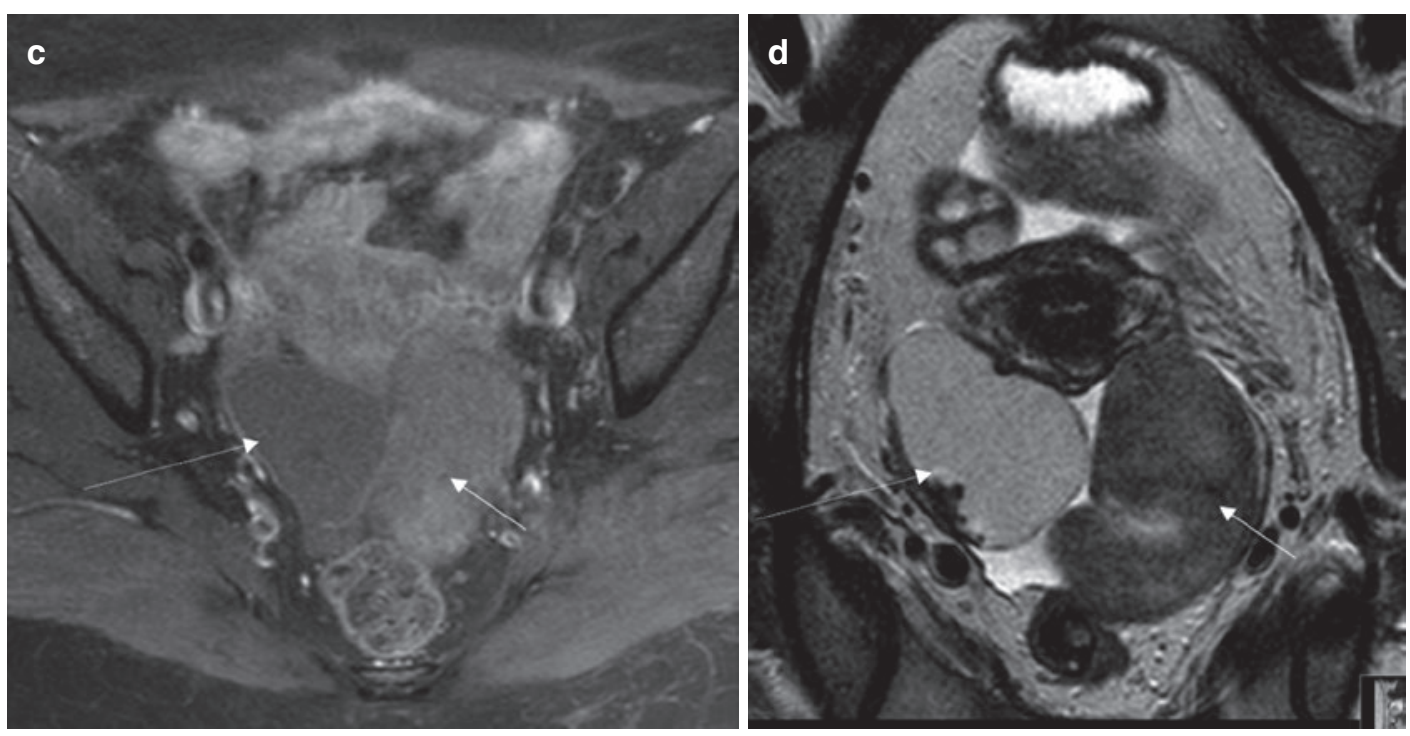

Fig. 19 (continued)

\subsubsection{Mature Teratoma}

Mature teratomas are the most common ovarian neoplasm in women under 45 years of age, and account for up to $70 \%$ of tumors in females less than 19 years of age (Koonings et al. 1989). In a large series it constitutes $10.6 \%$ of adnexal masses (Timmerman et al. 2016). Ovarian teratomas derive from germ cells and are classified into three main categories, among which the mature cystic teratomas account for $99 \%$. Less common types of mature teratomas are the monodermal teratomas, which include struma ovarii and carcinoid tumors. Monodermal teratomas are not cystic but contain primarily solid structures. Mature cystic teratomas typically contain lipid material consisting of sebaceous fluid within the cyst cavity or adipose tissue within the cyst wall or the dermoid plug.

\subsubsection{Mature Cystic Teratoma}

Mature cystic teratomas or dermoid cysts are composed of mature tissue from at least two of the three germ cell layers: ectoderm, mesoderm, and endoderm. They are typically unilateral lesions, with only $10-15 \%$ of mature cystic teratomas found in both ovaries (Kurman et al. 2011).
In the vast majority (88\%), mature teratomas are unilocular cystic lesions filled with sebaceous material. A protuberance, the Rokitansky nodule, or dermoid plug projects into the cavity and is the hallmark of mature teratomas. It contains a variety of tissues, often including fat and calcifications, which represent teeth or abortive bone. Fat is detected in over $90 \%$, teeth in $31 \%$, and calcifications in the wall in 56\% (Buy et al. 1989).

A minority of mature cystic teratomas will demonstrate no fat or only small foci of fat within the wall or the Rokitansky nodule (Yamashita et al. 1994). Yamashita et al. reported that $15 \%$ of mature teratomas did not show fat within the cystic cavity. Approximately half of these cases displayed small amounts of fat within the wall of the teratoma or the dermoid plug. In $8 \%$ of benign teratomas, no fat could be detected (Yamashita et al. 1994).

Mature teratomas are usually asymptomatic and tend to grow slowly. This is why watchful waiting is warranted in older females presenting with lesions smaller than $5 \mathrm{~cm}$ in size (Caspi et al. 1997). Complications encountered are malignant degeneration and rupture, and in up to $16 \%$ torsion (Rha et al. 2004). Malignant degeneration is extremely 
rare and associated with large size $(>10 \mathrm{~cm})$ and postmenopausal age. Rupture of a mature teratoma can cause acute abdomen due to granulomatous peritonitis caused by leakage of the fatty contents (Rha et al. 2004). Rarely, giant teratomas are found occupying the pelvis and abdomen. Recently ovarian teratoma-associated anti-NDMDAR encephalitis has been described. It is a potentially fatal disease in young women who clinically present with encephalitis caused by an underlying mostly with mature teratoma (Acien et al. 2014).

\section{Imaging Findings}

Sonographic assessment of mature cystic teratomas may be limited by its variety of appearance. At CT and MRI, however, the diagnosis of fat within a cystic mass is pathognomonic for a mature cystic teratoma (Figs. 20 and 21). The fatty elements display characteristic low CT attenuation $(-20$ to $-120 \mathrm{HU})$. Another typical feature on CT is the presence of calcifications within the cyst wall or the dermoid plug (Fig. 20).
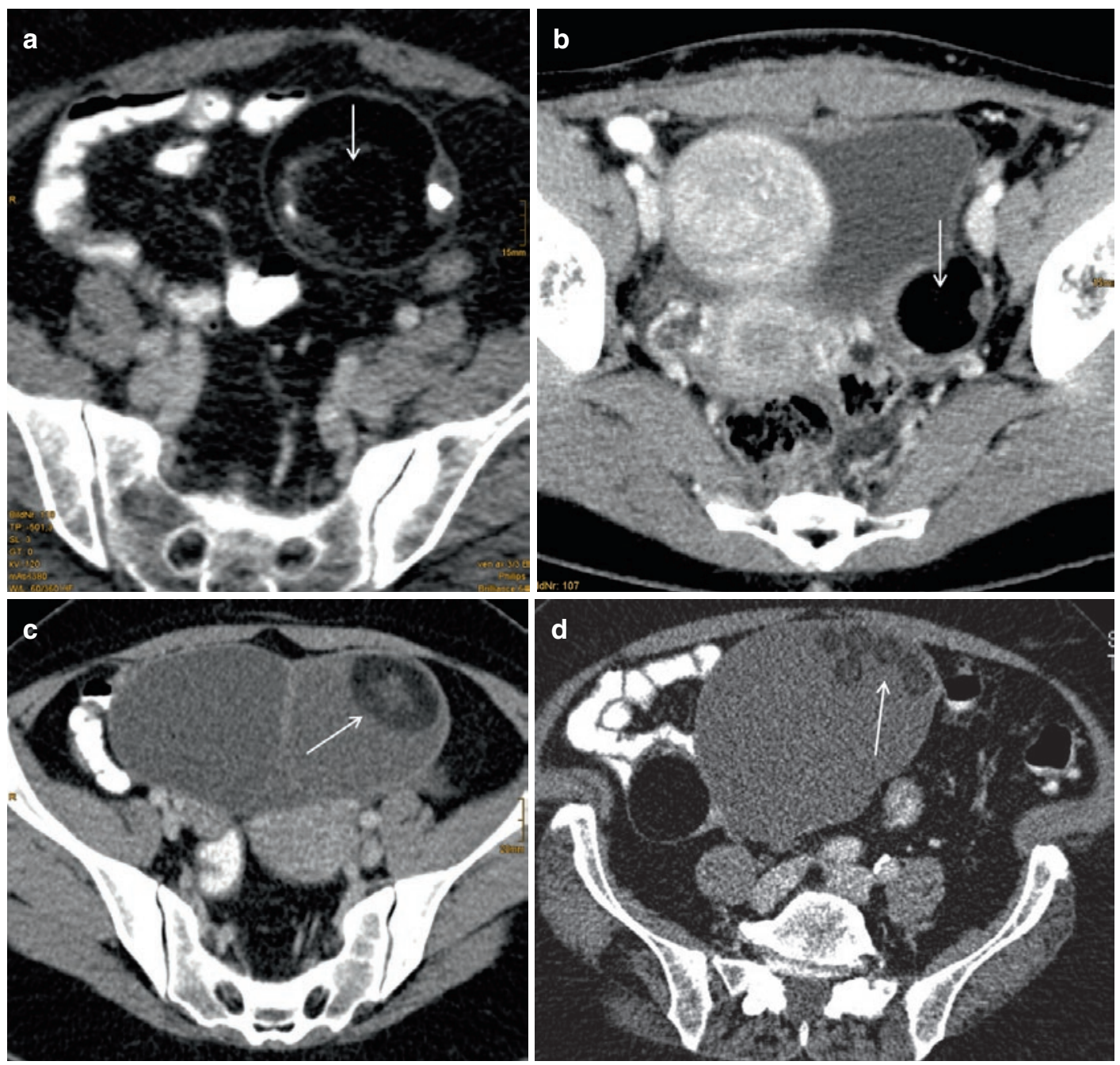

Fig. 20 A spectrum of CT findings in mature cystic teratomas in four different patients (a-d) is displayed. The arrow indicates fat as pathognomonic finding in this tumor type 


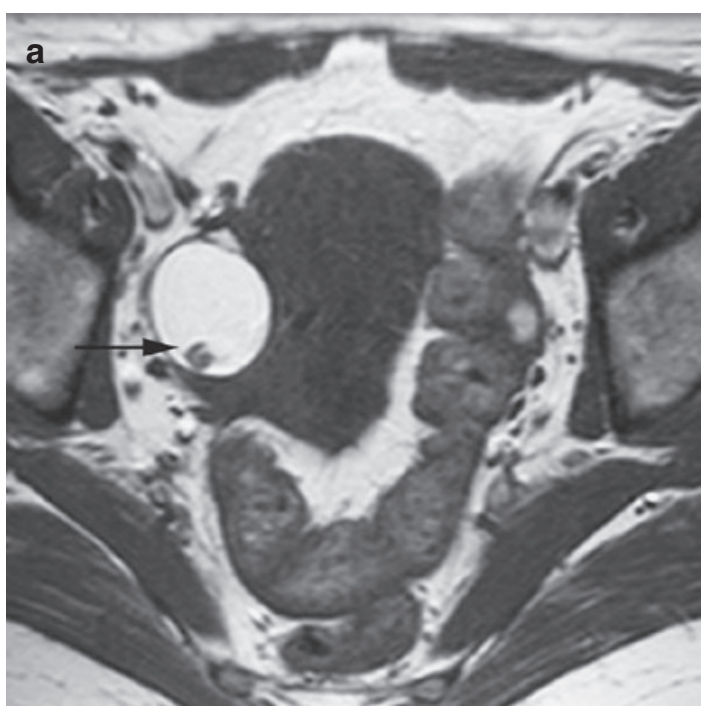

Fig. 21 Typical findings of a mature cystic teratoma on MRI. A 44-year-old woman complaining about irregular menstrual cycle and a suspicious adnexal mass at transvaginal ultrasound. Axial T1-weighted image (a) and contrast-enhanced T1-weighted image with fat suppression (b) at the acetabular level. The cystic structure of the

MRI findings include a round or oval, sharply delineated lesion with high SI on T1-weighted images, and loss of signal on the fat-saturated T1-weighted images, or signal loss in the out of phase chemical shift sequence. This fatty content may display a broad spectrum of appearance, including a fat-filled cavity, foci of fat within the lesion or its wall, and a fat-fluid interface often representing a floating mass of hair.

On T2-weighted images, the signal may be variable, but it tends to be similar to subcutaneous fat. Furthermore, chemical shift artifacts in the frequency-encoding direction can be observed, which confirms the presence of fat and differentiates it from hemorrhage. Calcification in the wall of or in the dermoid plug will often be missed on MRI due to the low SI on T1- and T2-weighted images. Caution is warranted in DWI because it may be misleading due to restricted diffusion (Forstner et al. 2016).

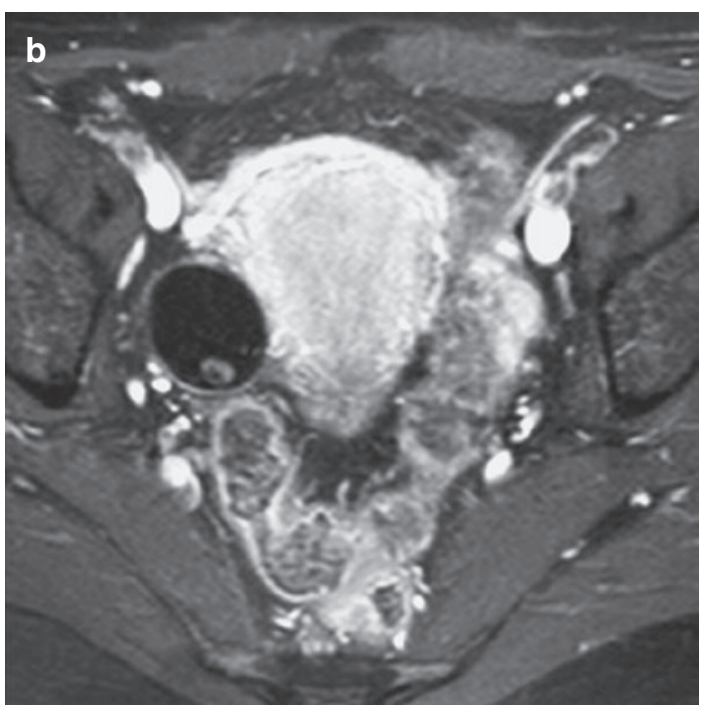

right ovary demonstrates hyperintense content with a round nodule in the lower part of the cyst (arrow) (a). The hypointense content after fat suppression (b) confirms the fatty nature of the cyst. At pathology, the round nodule corresponded to a hair ball within a mature cystic teratoma

\section{Differential Diagnosis}

Although hemorrhagic lesions including endometrioma, hemorrhagic cysts, and neoplasm may appear similar on the T1-weighted images and T2-weighted images, fat-suppressed or chemical shift images are most reliable for the differentiation of fat from hemorrhage.

When no or only small amounts of fat are present $(8 \%)$, teratomas are not distinguishable from benign cystic ovarian tumors or ovarian cancer (Fig. 22) (Rha et al. 2004).

Capsule penetration typically arising from the dermoid plug is a sign for malignant transformation of a mature teratoma (Rha et al. 2004). The rare liposarcoma or immature teratoma may contain fat and thus may be indiscernible from a teratoma. Immature teratomas, however, are extremely rare and occur in the first two decades of life. They may occur in association with an ipsilateral teratoma in $26 \%$ and a contralateral teratoma in $10 \%$. However, at the time of presentation they are usually very 

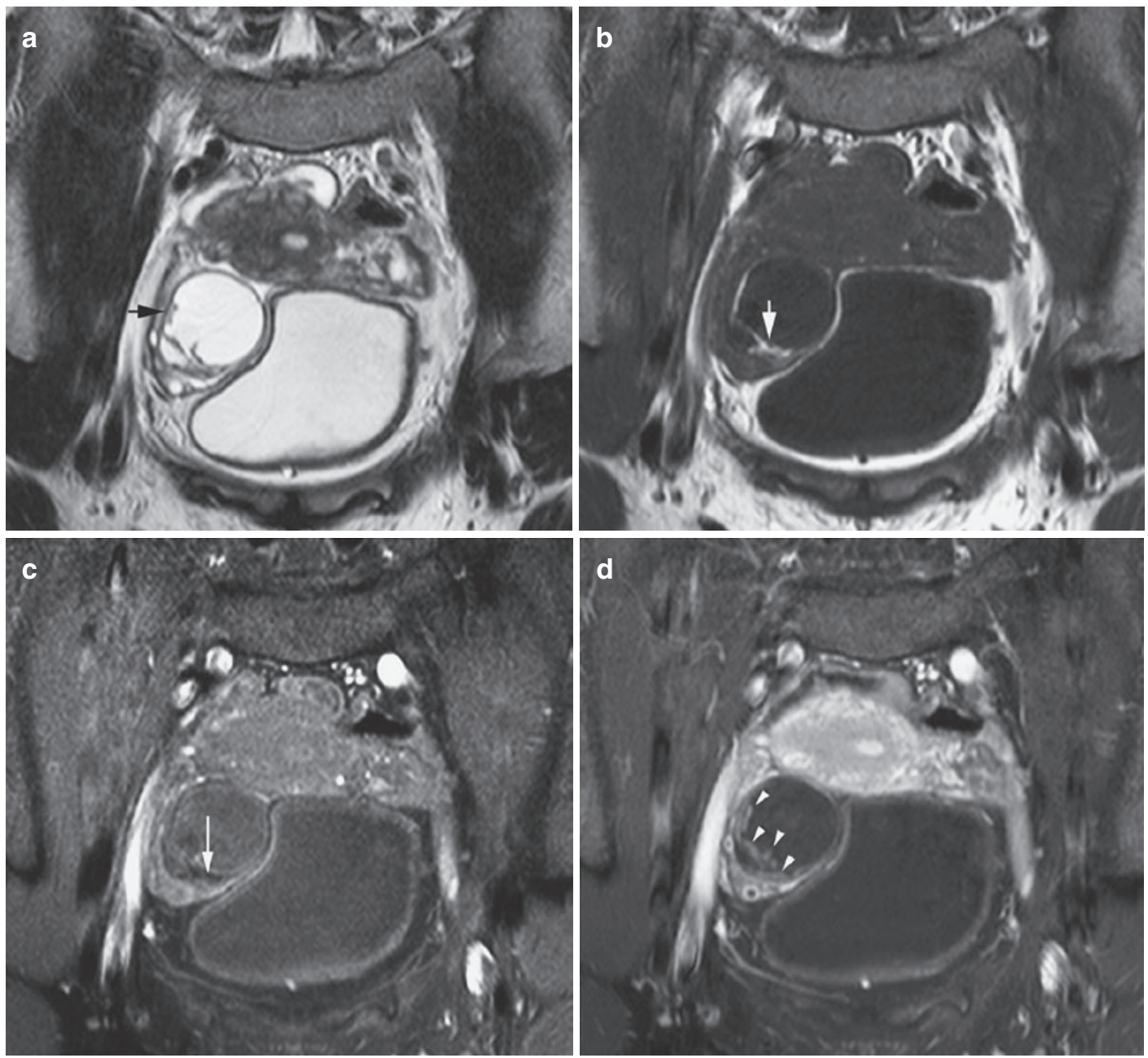

Fig. 22 Mature cystic teratoma with little fat. MRI was performed for further characterization of a sonographically suspicious cystic and solid mass in a 31-year-old woman. Coronal T2-weighted image (a), T1-weighted image (b), T1-weighted image with fat suppression (c), contrast-enhanced T1-weighted image with fat suppression (d). A multilocular mass with irregular wall thickening (arrow) (a) arising from the right adnexa is demonstrated. Coronal native T1-weighted image (b) confirms the cystic nature of the mass. A linear hyperintense portion is located at the lower part of the cyst (white

arrow). The corresponding coronal fat-suppressed T1-weighted image (c) shows vanishing of the hyperintense linear part confirming the presence of a small amount of fat in the linear hypointense lower portion of the cyst (white arrow). The contrast-enhanced fatsuppressed T1-weighted image (c) shows enhancement of an irregular wall and septa (arrowheads) corresponding to the Rokitansky nodule of a mature cystic teratoma with little fat. Contrast enhancement of the mural protrusion of a mature cystic teratoma can be mistaken for ovarian cancer 
large, are predominantly solid or cystic and solid, and contain only few foci of fat (Heifetz et al. 1998).

\subsubsection{Monodermal Teratoma}

Monodermal teratomas are composed mainly of one tissue type. They include struma ovarii, ovarian carcinoid tumors, and tumors with neural differentiation.

Struma ovarii is the most common type and accounts for $3 \%$ of all mature teratomas (Matsuki M 2000). It consists predominantly or solely of mature thyroid tissue. A mixed morphology with acini filled with thyroid colloid, hemorrhage, fibrosis, and necrosis is found. Rarely struma ovarii may produce thyrotoxicosis.

Carcinoid tumors are frequently associated with a mature cystic teratoma or a mucinous ovarian tumor. Unlike most cystic teratomas, they are predominantly found in postmenopausal women. The course is usually benign; there is a small subset of carcinoid tumors and of struma ovarii that are malignant. Carcinoid syndrome is uncommon.

\section{Imaging Findings}

On CT and MRI, a struma ovarii displays as a heterogeneous complex mass (Fig. 23). They present as cystic lesions or with a multilocular appearance with loculi displaying high signal intensity on $\mathrm{T} 1$ and $\mathrm{T} 2$, some with low signal intensity on T1- and T2-weighted images on MR. Fat is not seen in struma ovarii. Carcinoid tumors are solid tumors indistinguishable from solid ovarian malignancies.

\subsubsection{Benign Sex Cord-Stromal Tumors}

Sex cord-stromal tumors include neoplasms that are composed of granulosa cells, theca cells, and their luteinized derivates, including Sertoli cells,
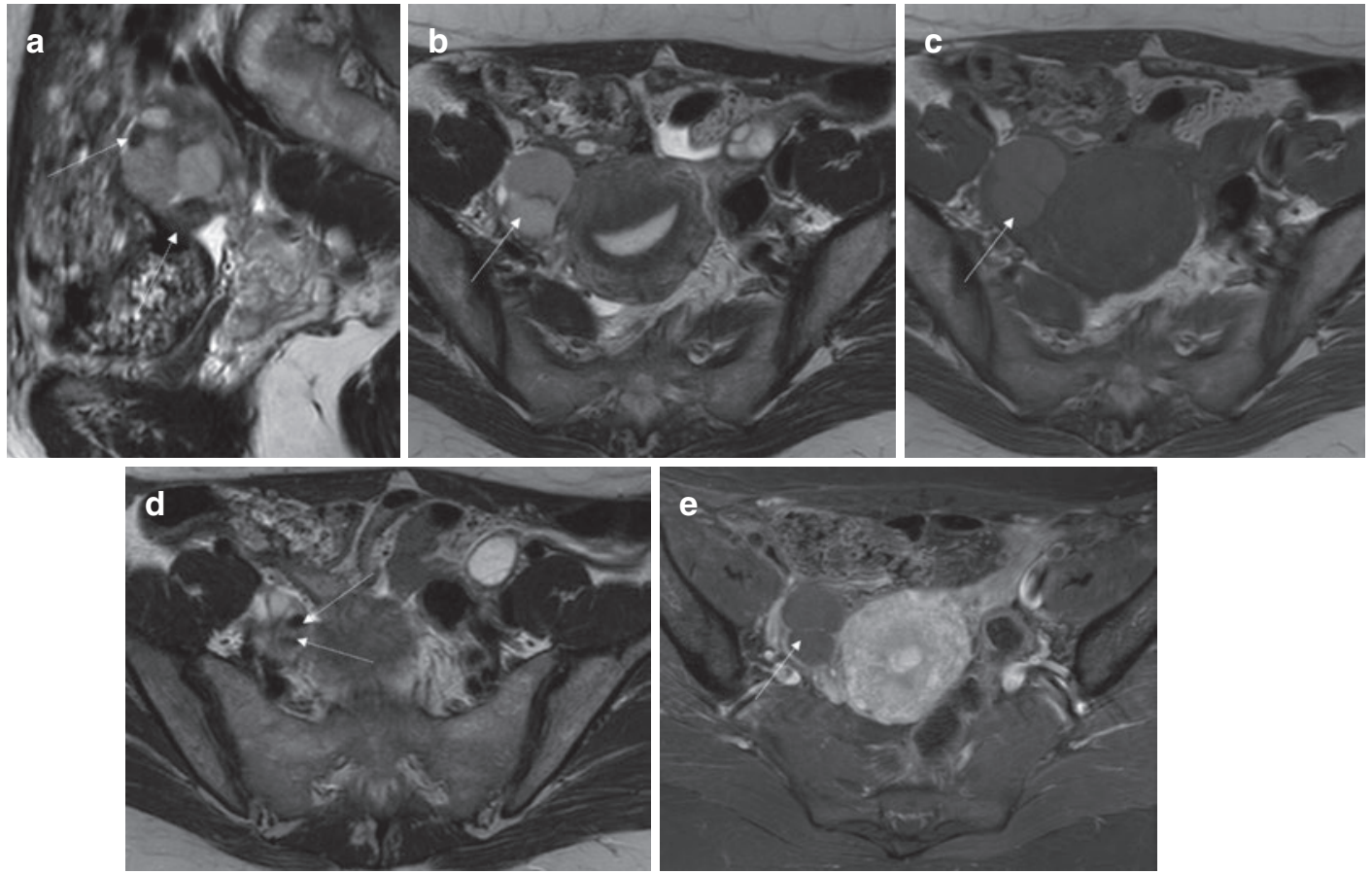

Fig. 23 Struma ovarii in a 49-year-old patient. (a) Sagittal T2-weighted image; (b) axial T2-weighted image; (c) axial T1-weighted image; (d) axial T2-weighted image; (e) axial fat-suppressed gadolinium-enhanced T1-weighted image. T1- and T2-weighted images show a multiloculated cystic mass in the right ovary, with locules of variable signal intensity. There are locules showing high signal both on T1- and T2-weighted images and low signal fat-suppressed gadolinium-enhanced T1-weighted images that are consistent with fat (arrows). Note the typical locules with low T2-weighted signal due to the presence of colloid content (dashed arrows) 
Leydig cells, and fibroblasts of gonadal stromal origin (Young and Scully 2002). They present rare tumors that differ from epithelial neoplasm due to their hormonal activity (Horta and Cunha 2015). Sex cord-stromal tumors have recently been classified as pure stromal tumors, pure sex cord tumors, and mixed sex cord-stromal tumors (Kurman et al. 2014).

Among the pure stromal tumors fibroma and thecoma account for the vast majority of benign sex cord-stromal tumors.

\subsubsection{Fibroma and Thecoma}

Fibromas and thecomas are solid ovarian tumors accounting for $3-4 \%$ of all ovarian tumors and $10 \%$ of solid adnexal masses. They are typically unilateral $(90 \%)$ and occur in peri- and postmenopausal age women. Histologically they present the majority of sex cord-stromal tumors.

Fibromas are composed mostly of fibroblasts and spindle cells and abundant collagen contents. Fibromas are not hormonally active. The triad of an ovarian fibroma, ascites, and pleural effusion constitutes the benign Meigs syndrome, which can be associated with elevated CA-125 levels (Timmerman et al. 1995). In basal cell nevus syndrome, numerous basal cell carcinomas are associated with abnormalities of bones, eyes, brain, and tumors, including ovarian bilateral fibromas.

Thecomas are composed of thecal cells with abundant and varying amounts of fibrosis and may rarely contain dense amorphous calcifications. Unlike fibromas, $60 \%$ of thecomas have estrogenic activity and may present with uterine bleeding. Furthermore, in more than $20 \%$, endometrial carcinomas occur concomitantly (Outwater et al. 1997).

\section{Imaging Findings}

Small fibromas and thecomas are solid tumors with imaging features similar to nondegenerative uterine leiomyomas on CT and MRI (Figs. 24 and 25). They display intermediate to low SI on T1-weighted images and typically very low SI or low SI with intermediate SI on the T2-weighted images on MRI (Fig. 25). Large lesions may have an inhomogeneous architecture with areas of high signal intensity within the solid low signal intensity lesion, representing edema or cystic degeneration (Chung et al. 2015). They had in $40 \%$ atypical features with mixed solid/cystic and predominantly cystic morphology and displayed high SI on T1 and T2WI in almost half of the cases. Small amounts of ascites were a common finding (Chung et al. 2015). Another feature is dense amorphous calcifications, which are

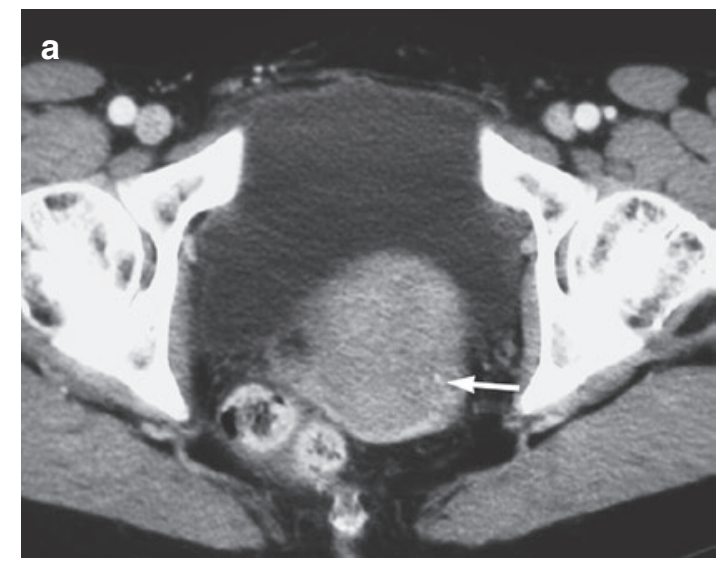

Fig. 24 Ovarian fibroma on CT. Transaxial pelvic CT at the uterine level (a) and above (b) in a 55-year-old woman with abdominal fullness. A large lesion (asterisk) is found in the mid pelvis above the level of the uterus and bladder (b). It is well demarcated and displays a slightly inhomo-

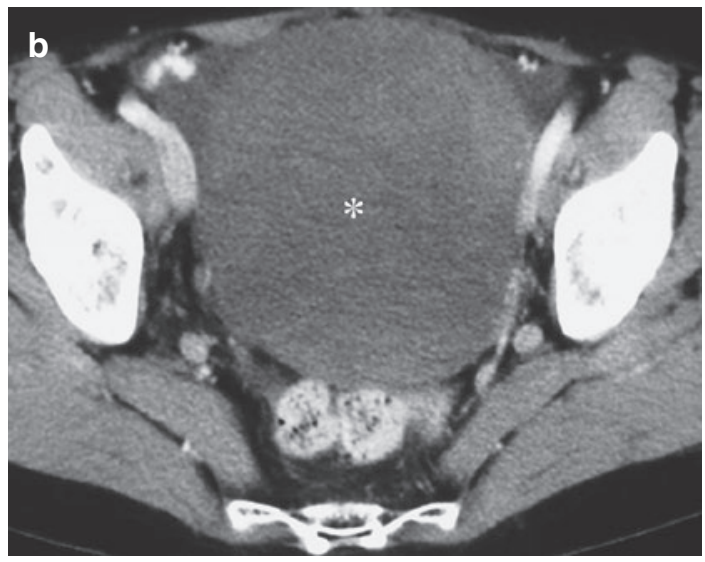

geneous solid structure. Contrast enhancement is distinctly less than that of the myometrium (arrow). No calcifications were found throughout the lesion. Minimal ascites was seen. Histopathology revealed a $9 \mathrm{~cm}$ fibroma of the left ovary 

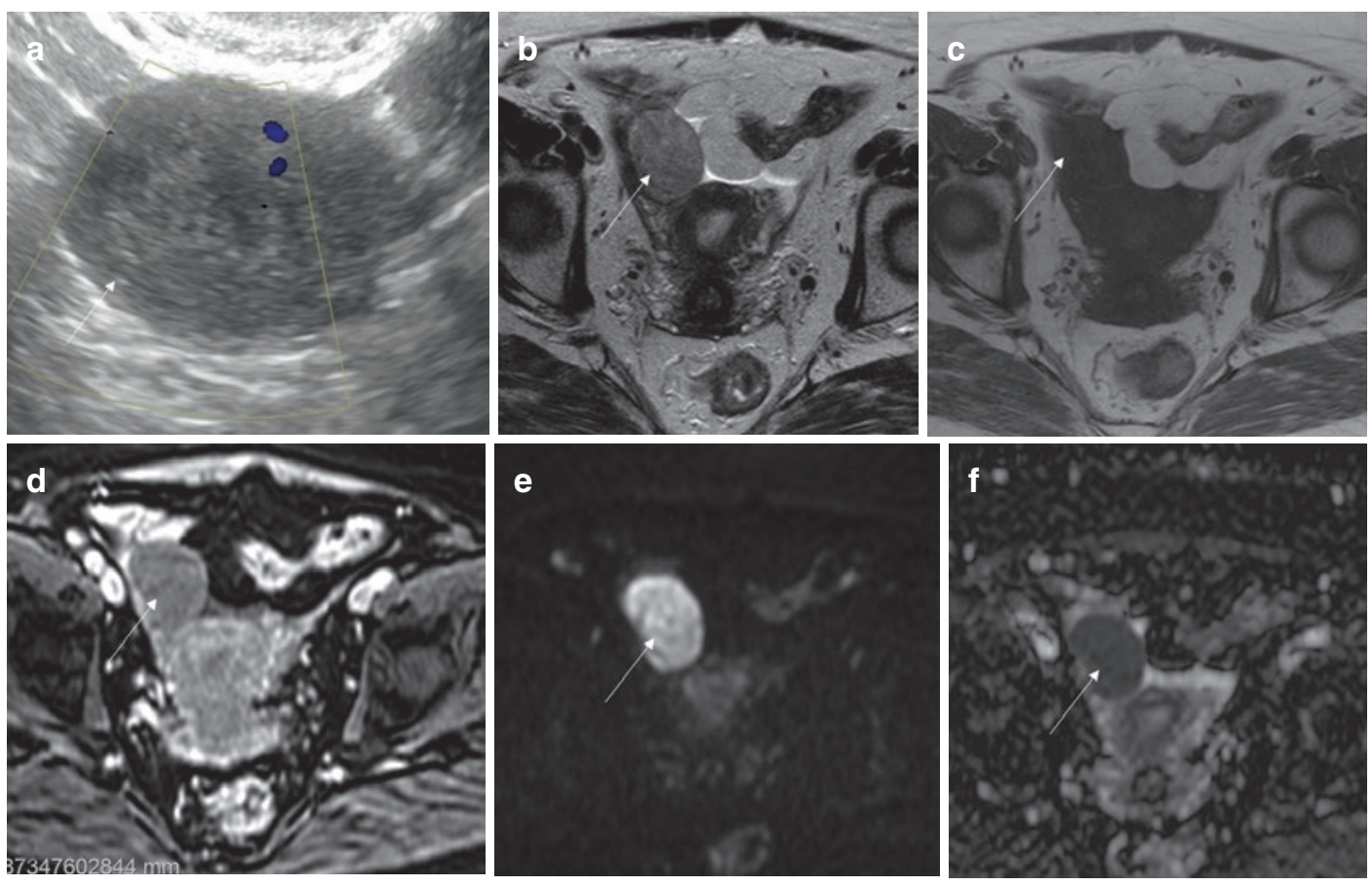

Fig. 25 A 67-year-old patient with a right ovarian thecoma. (a) Transvaginal ultrasound image; (b) axial T2-weighted image; (c) axial T1-weighted image; (d) fatsaturated axial gadolinium-enhanced T1-weighted image; (e) axial diffusion-weighted image $\left(b=1000 \mathrm{~s} / \mathrm{mm}^{2}\right)$; (f)

easily detected on CT. Fibromas and thecomas tend to show mild or delayed contrast enhancement and typically a type 1 time intensity pattern. They may also display a type 2 time intensity curve. Rarely cellular fibromas may be found, which tend to be well vascularized and have a low malignant potential (Horta and Cunha 2015) (Fig. 26). Low SI on the high $b$ value in DWI allows the exclusion of malignancy in solid adnexal masses (Thomassin-Naggara et al. 2009). Ascites may be present and even large amounts are no sign of malignancy.

\subsubsection{Sclerosing Stromal Tumor}

Sclerosing stromal tumor of the ovary is a rare subtype of sex cord-stromal tumors. It is a benign tumor affecting most commonly young girls and women younger than 30 years of age, which is much earlier in other stromal tumor types (Matsubayashi et al. 1999; Palmeiro et al. 2016). Macroscopically, these tumors display peripheral axial ADC map. Ultrasound image shows a right homogeneous vascularized solid mass. T2-weighted image shows a solid intermediate signal lesion. The lesion displays low signal intensity on T1-weighted image, only moderate contrast uptake, and restricted diffusion (arrows)

edematous ovarian cortical stroma surrounding nodular highly vascular cellular components (Matsubayashi et al. 1999; Palmeiro et al. 2016). Some of these tumors may have an estrogenic effect and rarely androgenic effects, which cause prolonged menstrual irregularities or pelvic pain. Ascites may be rarely found.

\section{Imaging Findings}

Sclerosing stromal tumors of the ovary tend to be unilateral well-encapsulated multiloculated cystic or heterogeneous ovarian lesions with a characteristic centripetal contrast enhancement pattern (Fig. 27). On T1- and T2-weighted images, a thin low-intensity rim representing a capsule is seen. On T2-weighted images, in the periphery an irregular low-signal-intensity rim is found adjacent to a very bright more central portion, which has a nodular appearance. On dynamic imaging, the arterial phase shows minimal contrast uptake, whereas in the venous 

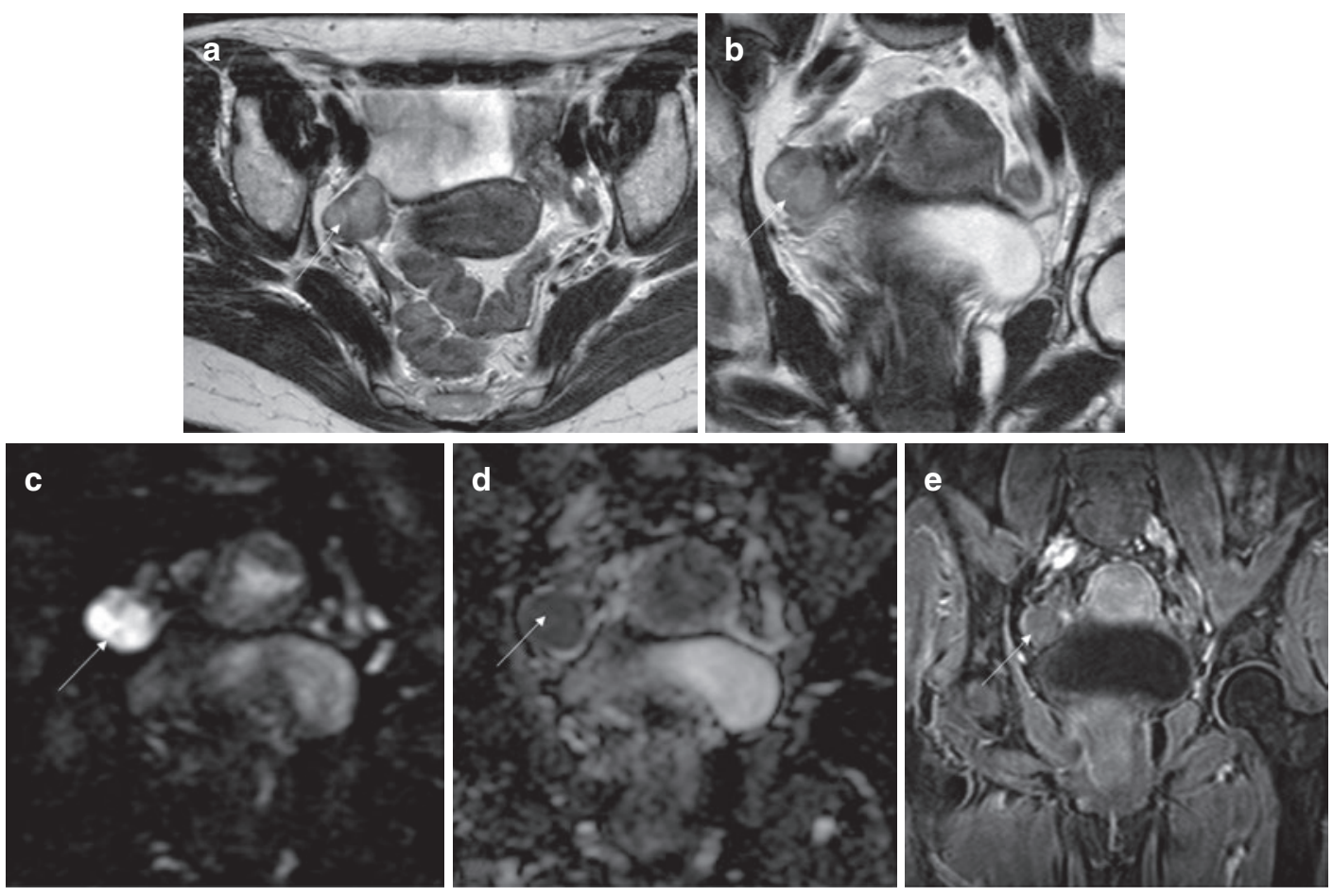

Fig. 26 Cellular fibroma of the right ovary in a 60-yearold patient. (a) Axial T2-weighted image; (b) axial oblique T2-weighted image; (c) axial oblique diffusionweighted image $\left(b=1000 \mathrm{~s} / \mathrm{mm}^{2}\right) ;(\mathbf{d})$ axial oblique ADC

phase high contrast uptake and a pseudolobular pattern are seen. Slow centripetal progression on delayed images has been described as a typical imaging feature (Matsubayashi et al. 1999).

\subsubsection{Brenner Tumors}

Brenner tumors are rare ovarian tumors that occur at a mean age of 50 years. Brenner tumors constitute $1-3 \%$ of ovarian tumors. They are mostly benign, with less than $2 \%$ demonstrating borderline or malignant transformation. They are typically small, solid, unilateral ovarian tumors, with $60 \%$ of these tumors found fewer than $2 \mathrm{~cm}$ in size. Extensive calcification may be found. The vast majority is discovered incidentally in pathologic specimen of the adnexa. Brenner tumors rarely produce estrogen, and then they may be associated with endometrial thickening (Moon et al. 2000). If cystic components are found in Brenner tumors, they may be associated map; (e) fat-saturated axial oblique gadolinium-enhanced T1-weighted image. On T2-weighted images the intermediate signal intensity of the cellular fibromas is shown (a) and (b)

with cystadenomas (Seidman et al. 2002). Up to $20 \%$ of Brenner tumors are associated with mucinous cystadenomas or other epithelial neoplasm (Fig. 28).

\subsubsection{Imaging Findings}

The typical finding of Brenner tumors is a small solid tumor that displays very low SI on the T2-weighted images (Moon et al. 2000). Dense amorphous calcifications in a small solid ovarian tumor are a typical CT finding. In one series of eight Brenner tumors, the mean size was $11.5 \mathrm{~cm}$, and tumors displayed a mixed solid and cystic appearance in half of the cases, which mimicked ovarian cancer (Moon et al. 2000). The combination of a multiseptate ovarian tumor with a solid part displaying extensive calcifications on CT or very low SI on MRI may suggest the diagnosis of a collision tumor of Brenner tumor and a cystic ovarian neoplasm, e.g., cystadenoma (Fig. 28). 

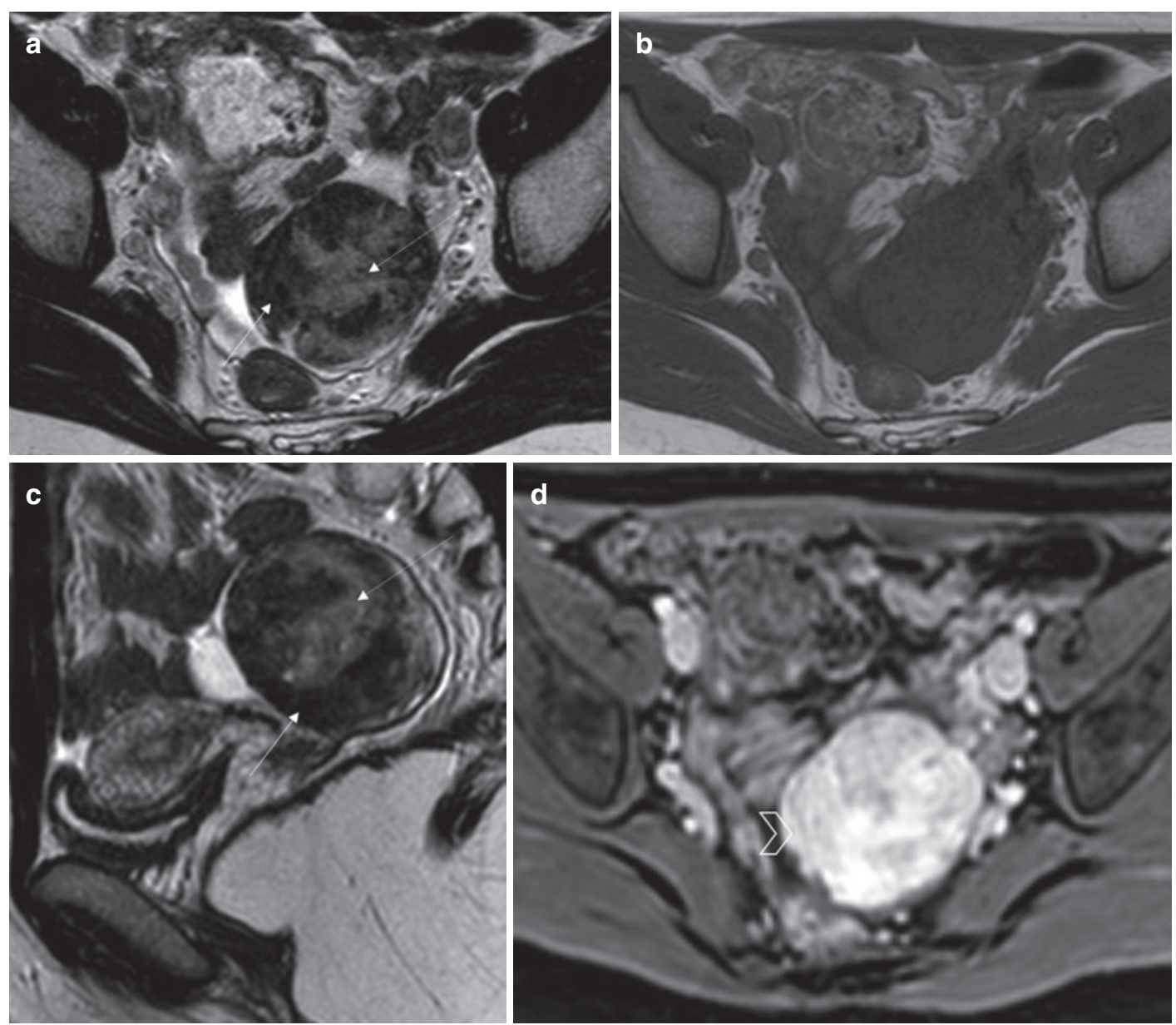

Fig. 27 Sclerosing stromal tumor of the left ovary in an 18-year-old patient. (a) Axial T2-weighted image; (b) axial T1-weighted image; (c) sagittal T2-weighted image; (d) axial fat-suppressed gadolinium-enhanced T1-weighted image. T2-weighted images show a left ovarian mass with

\subsubsection{Differential Diagnosis}

The solid morphology and the very low SI on the T2WI of fibromas and thecomas are fairly characteristic. Pedunculated uterine leiomyomas and leiomyomas of the broad ligaments can display similar imaging characteristics. Subserosal pedunculated leiomyomas can be discriminated by the bridging vascular sign. Unilateral or bilateral ovarian leiomyomas are extremely rare and a pseudolobular spoke-wheel pattern that is characterized by outer hypointense solid component (arrows) and a central intermediate signal intensity area (dashed arrows). The outer solid component shows avid enhancement after gadolinium administration (arrowhead)

cannot be reliably differentiated by imaging. High-contrast media uptake in such a lesion might suggest ovarian leiomyoma (Yoshitake et al. 2005). Fibromas with large central necrotic area mimic malignant ovarian masses, especially Krukenberg tumors. DWI and most importantly DCE will allow the differentiation in the majority of cases (see Chap. 66). Dense amorphous calcifications in CT support also the diagnosis of 

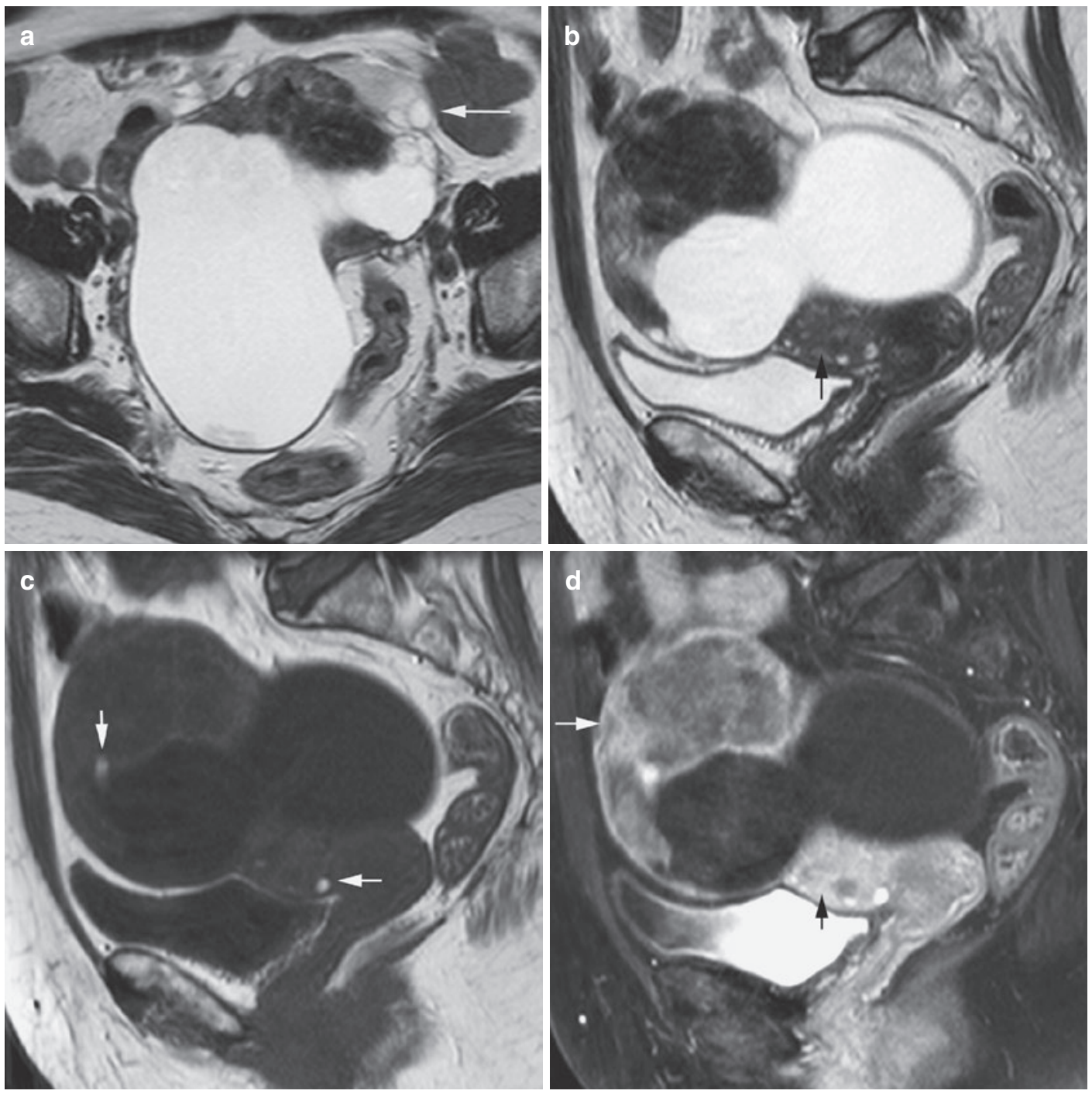

Fig. 28 Large Brenner tumor with mucinous portion. A 75-year-old woman presenting with a large suspicious pelvic mass at ultrasound and moderate increase in CA-125 level. Axial T2-weighted image (a), sagittal T2-weighted image (b), sagittal T1-weighted image (c), sagittal T1-weighted image with fat suppressed. (d). A cystic mass with a solid hypointense anterior portion is demonstrated (a). Ovarian parenchyma with two follicles is seen at the left anterior side (arrow). The small uterus (arrow) is identified below the left ovarian mass (b). The interface between the cystic and solid portion of the mass is regular. Sagittal T1-weighted image (c)

shows absent blood within the cyst except for two blood vessels seen within the solid portion of the mass and the anterior myometrium (arrows). At the same level as (b) and (c) contrast-enhanced T1-weighted image with fat suppression (d) shows heterogeneous decreased contrast enhancement of the solid portion of the cyst (white arrow) compared to the strongly enhancing myometrium (black arrow). Histology after hysterectomy and bilateral oophorectomy diagnosed a benign Brenner tumor of the left ovary with an associated benign mucinous portion 
stromal tumors. Small calcified solid tumors favor the diagnosis of Brenner tumors. Unlike in benign stromal tumors, calcifications in ovarian cancer tend to be small punctuating foci, the socalled psammoma bodies.

Sclerosing stromal cell tumors have a pathognomonic centripetal contrast media uptake. Morphologically, they may resemble Krukenberg tumors, but young age is characteristic of this entity.

\section{$5 \quad$ Functioning Ovarian Tumors}

Clinical and imaging findings may lead to the diagnosis of a functioning ovarian tumor.

The imaging findings comprise an ovarian mass, but also indirect findings as abnormalities of the uterus with uterine enlargement, a thickened endometrium in pre- and postmenopausal women, abnormal bleeding, features of virilization, or endocrinologic symptoms (Tanaka et al. 2004).

Sex cord-stromal tumors account for the majority of functioning ovarian tumors. These benign masses as well as neoplasms of low malignant potential account for the majority of estrogen-producing tumors. Granulosa cell tumors and thecomas are the most common estrogen-producing tumors (Fig. 29). Some mucinous cystadenomas and rarely ovarian cancer and metastases may also produce estrogens (Young and Scully 2002). In the majority of women of reproductive age, virilization is associated with the benign polycystic ovaries syndrome (PCOS). Virilizing ovarian tumors are rare and present mostly solid ovarian tumors (Fig. 30). Sertoli-Leydig cell tumors are typically found in young women and account for two-thirds of these tumors causing hirsutism or virilization. In middle-aged women, steroid cell tumors can cause virilization and/or Cushing's syndrome. Furthermore, rarely granulosa cell tumors, Brenner tumors, and thecomas may also have virilizing effects.

Thyroid hormones are typically produced in struma ovarii in subclinical levels. Hyperthyreosis seems to be present in only $25 \%$, and thyrotoxicosis occurs in only $5 \%$ of patients with struma ovarii (Young and Scully 2002). Primary carcinoids of the ovary are rarely associated with carcinoid syndrome. Metastatic carcinoids involving the ovary, however, are associated with carcinoid syndrome in $50 \%$ of cases. Benign and malignant mucinous ovarian tumors may produce gastrin within the cyst wall and present clinically with Zollinger-Ellison syndrome (Garcia-Villanueva et al. 1990).
Fig. 29 Granulosa cell tumor. A 52-year-old female with a history of hysterectomy and unilateral oophorectomy for granulosa cell tumors several years before. A solid and cystic pelvic tumor with irregular margins displacing bowel loops is seen at the acetabular level. From imaging, it cannot be differentiated from an ovarian cancer

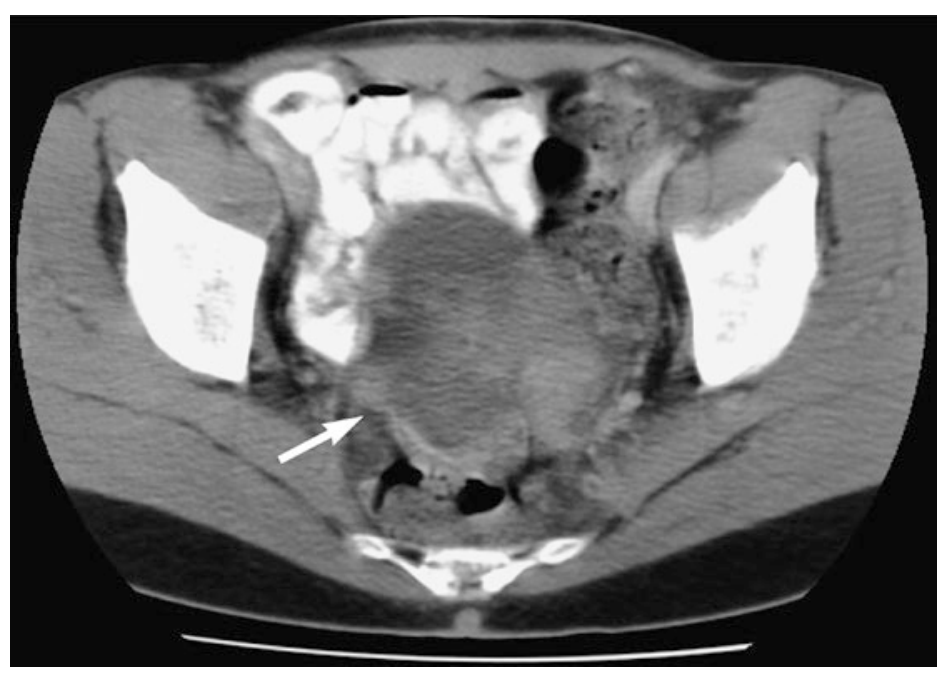



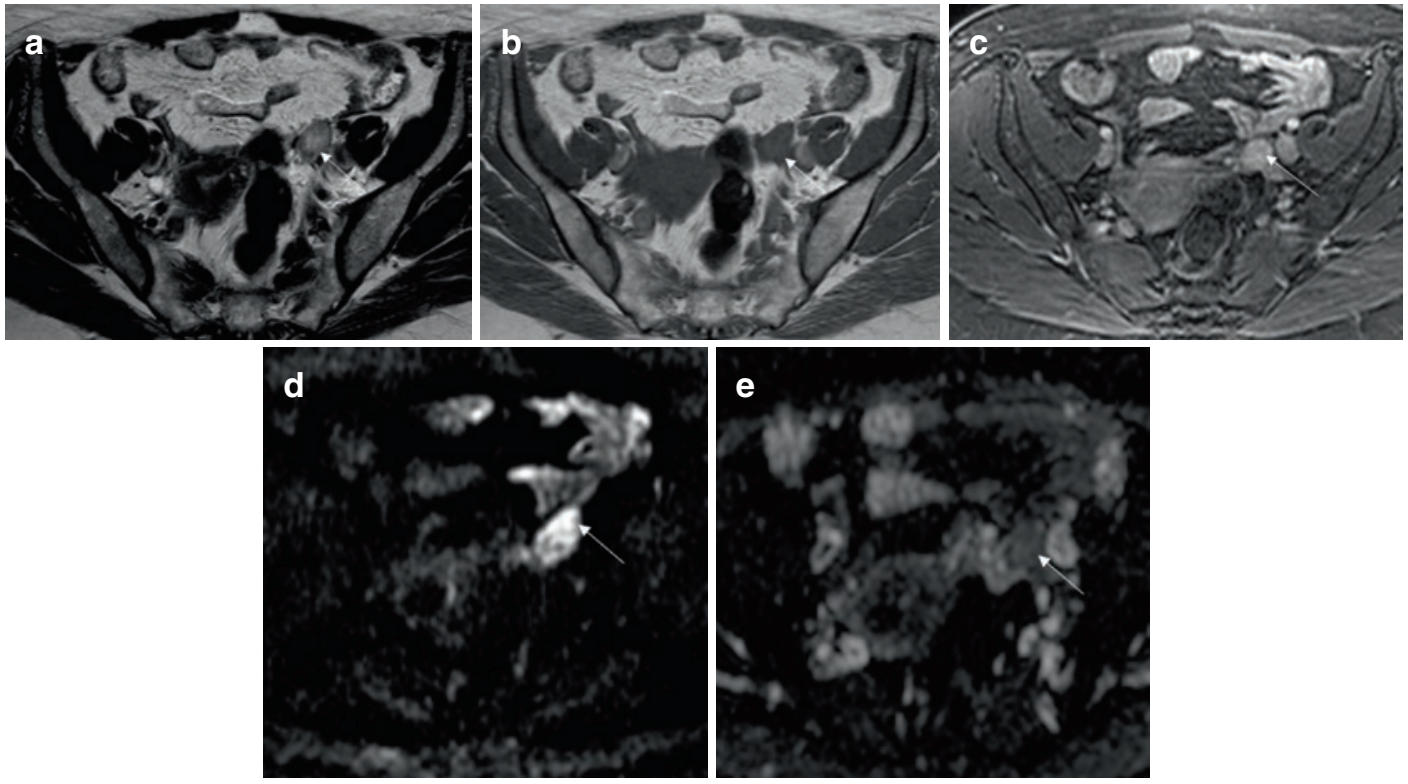

Fig. 30 A 63-year-old patient with a left steroid cell tumor (former stromal luteoma) presenting with hirsutism. (a) Axial T2-weighted image; (b) axial T1-weighted image fat; (c) saturated axial gadolinium-enhanced T1-weighted image; (d) axial diffusion-weighted image

\section{Ovarian Tumors in Children, Adolescents, and Young Women}

The majority of ovarian masses in children older than 9 years and young women are benign and include follicular cysts and mature cystic teratomas, with fewer than $5 \%$ of ovarian malignancies occurring in this age group. However, lesions with complex architecture should be carefully assessed, as $35 \%$ of all malignant ovarian neoplasms occur during childhood and adolescence. This is especially true for children younger than 9 years, where approximately $80 \%$ of ovarian neoplasms are malignant (Norris and Jensen 1972). A solid ovarian mass in childhood should also be considered malignant until proven otherwise by histology (Laufer 2017). Differential diagnosis includes dysgerminoma, neuroblastoma rhabdomyosarcoma, lymphoma, and nongenital tumors in the pelvis. Some ovarian neoplasms occurring in this age group excrete protein tumor markers, which may aid in diagnosis and follow-up. They include alpha-fetoprotein, $\left(b=1000 \mathrm{~s} / \mathrm{mm}^{2}\right) ;(\mathbf{e})$ axial ADC map. Images show a small solid tumor that on T2-weighted images is hyperintense to the ovarian stroma. There is enhancement of the lesion as well as restricted diffusion (arrows)

which is produced by endodermal sinus tumors, mixed germ cell tumors, and immature teratomas, lactate dehydrogenase, which is secreted by dysgerminomas, and human chorionic gonadotropin, which is elevated in pregnancy and pregnancy-related tumors and in embryonal ovarian carcinomas (Laufer 2017). Torsion is a special problem in children and young adults presenting with an ovarian mass. Ovarian masses associated with torsion present benign cystic lesions (Fig. 31) with a size greater than $5 \mathrm{~cm}$ seem to be under a high risk for torsion (Cass et al. 2001). In children also normal ovaries may undergo torsion. Acute pelvic pain is the mainstay in the differential diagnosis of a torsed ovary; however, imaging findings may sometimes be subacute and misleading and simulate a malignant ovarian tumor.

Ovarian cysts are uncommon before puberty. Most of these are physiologic follicular cysts that will resolve spontaneously. Some ovarian cysts may be hormonally active and result in precocious pseudopuberty, e.g., in McCune-Albright syndrome (Frisch et al. 1992). Ovarian cysts are 

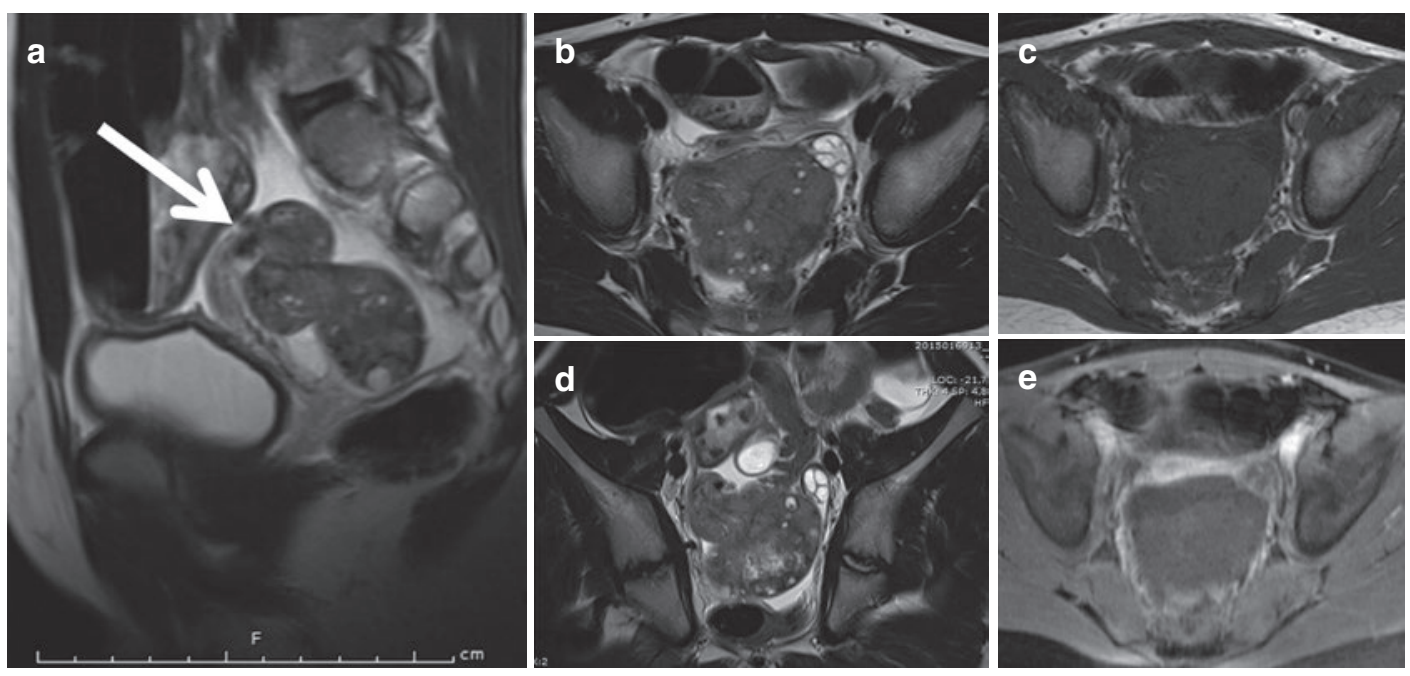

Fig. 31 Right ovarian torsion in a 10-year-old girl. Sagittal T2-weighted image (a), axial T2-weighted image (b), coronal T2-weighted image (c), axial T1-weighted image (d), axial T1-weighted image with FS (e). Common findings are somewhat nonspecific and include an adnexal mass that may be in the midline, rotated toward the contralateral side of the pelvis; deviation of the uterus to the side of the affected ovary (e); and ascites. An enlarged right ovary with peripheral cysts (up to $1 \mathrm{~cm}$ in diameter)

extremely common between puberty and 18 years of age. Most of these cysts are functional ovarian cysts and may attain a size of up to $8-10 \mathrm{~cm}$. In this age group, paraovarian or mesothelial cysts, hydrosalpinx, and obstructive genital lesions may also simulate cystic ovarian lesions. Germ cell tumors account for half to twothirds of the tumors in girls up to 18 years; they present $70 \%$ of ovarian tumors in the age between 10 and 30 years (van Winter et al. 1994). The vast majority is unilateral and present benign teratomas. Only $3 \%$ of ovarian germ cell tumors are malignant. Dysgerminomas account for approximately $50 \%$ of the malignant germ cell tumors in adolescents and young adults and are followed by endodermal sinus tumors (20\%) and immature teratomas (19\%) (van Winter et al. 1994). As in many ovarian malignancies, rapid growth is a typical finding; however, bilateral manifestation is more common in dysgerminomas than in other malignant germ cell tumors. Juvenile granulosa cell tumors are stromal cell tumors of low malignant potential, which occur before the age of 30 . and no evidence of blood flow is seen. A small amount of pelvic free fluid is also noted. A beaked protrusion at the periphery of the affected ovary, a finding consistent with engorged blood vessels (arrow) (a) and absence of enhancement (e) can be observed. At surgery, the ovary was hemorrhagic and necrotic, appearing with a $360^{\circ}$ twist of the pedicle. At pathologic analysis, a congested hemorrhagic ovary with no normal ovarian tissue was identified

Rarely, they develop before puberty and may become clinically apparent as precocious puberty. Immature teratomas are commonly associated with a mature teratoma; they comprise $1 \%$ of all teratomas and occur most commonly in the first two decades of life. Tumor markers are usually negative.

\section{$7 \quad$ Adnexal Masses in Pregnancy}

Adnexal masses have been reported to occur in 1-2\% of pregnancies (Chiang and Levine 2004). Most of these masses are found incidentally, present functional cysts and will disappear during the first 16 weeks of pregnancy (Hermans et al. 2003). The incidence of ovarian cancer associated with a persistent adnexal mass varies from $3 \%$ to $5.9 \%$. In a retrospective analysis of 60 adnexal masses during pregnancy, 50\% included mature cystic teratomas, $20 \%$ cystadenomas, $13 \%$ functional ovarian cysts, and $13 \%$ malignant tumors. Among the latter, six 
out of eight were tumors of low malignant potential, and all malignant lesions were FIGO stage IA tumors (Sherard et al. 2003). The management of an adnexal mass during pregnancy depends on the size, sonomorphologic criteria, and gestational age. MRI offers incremental benefit in diagnosing exophytic leiomyomas, atypical mature cystic teratomas, and in assessing distant spread in frankly malignant adnexal masses (Telischak et al. 2008). Well-vascularized adnexal masses that are pregnancy related include decidualized endometriomas and pregnancy luteomas. These may mimic malignancy, but clinical background combined with US and MRI may suggest the correct diagnosis and warrant a follow-up (Telischak et al. 2008; Tannus et al. 2009). Pain or an acute abdomen should alert to complications due to hemorrhage, rupture, and torsion of the adnexal mass or nongynecological pelvic conditions.

\section{References}

Acien P, Acien N, Ruiz-Macia E, Martin-Estefania C (2014) Ovarian teratoma-associated anti-NDMDAR encephalitis: a systematic review of reported cases. Orphanet J Rare Dis 9:157. doi:10.1186/s13023-014-0157-X

Buy JN, Ghossain MA, Moss AA et al (1989) Cystic teratoma of the ovary: CT detection. Radiology 171:697670. doi:10.1148/radiology.171.3.2717741.

Caspi B, Appelman Z, Rabinerson D et al (1997) The growth pattern of ovarian dermoid cysts: a prospective study in premenopausal and postmenopausal women. Fertil Steril 68:501-505

Cass DL, Hawkins E, Brandt ML et al (2001) Surgery for ovarian masses in infants, children, and adolescents: 102 consecutive patients treated in a 15 -year period. J Pediatr Surg 36:693-699. doi:10.1053/jpsu.2001.22939

Chiang G, Levine D (2004) Imaging of adnexal masses in pregnancy. J Ultrasound Med 23:805-819

Christensen JT, Boldsen JL, Westergaard JG (2002) Functional ovarian cysts in premenopausal and gynecologically healthy women. Contraception 66:153-157

Cho SM, Byun JY, Rha SE et al (2004) CT and MRI findings of cystadenofibromas of the ovary. Eur Radiol 14:798-804. doi:10.1007/s00330-003-2060-Z

Chung BM, Park SB, Lee JB, Park HJ, Kim YS, Oh YJ (2015) Magnetic resonance imaging features of ovarian fibroma, fibrothecoma, and thecoma. Abdom Imaging 40:1263-1272. doi:10.1007/s00261-014-0257-z

Clement PB (2002) Non-neoplastic lesions of the ovary. In: Kurman RJ (ed) Blaustein's pathology of the female genital tract. Springer Verlag, New York, pp 675-728
Corwin MT, Gerscovich EO, Lamba R, Wilson M, McGahan JP (2014) Differentiation of ovarian endometriomas from hemorrhagic cysts at MR imaging: utility of the T2 dark spot sign. Radiology 271:126132. doi:10.1148/radiol.13131394

Dias JL, Veloso Gomes F, Lucas R, Cunha TM (2015) The shading sign: is it exclusive of endometriomas? Abdom Imaging 40:2566-2572. doi:10.1007/ s00261-015-0465-1

Duijkers IJ, Klipping C (2010) Polycystic ovaries, as defined by the 2003 Rotterdam consensus criteria, are found to be very common in young healthy women. Gynecol Endocrinol 26:152-160. doi:10.1080/ 09513590903247824

Forstner R, Thomassin-Naggara I, Cunha TM et al (2016) ESUR recommendations for MR imaging of the sonographically indeterminate adnexal mass: an update. Eur Radiol Oct 21 [Epub ahead of print] Erratum in: Eur Radiol. 2016 Dec 5. doi:10.1007/s00330-016-4600-3

Foshager MC, Hood LL, Walsh JW (1996) Masses simulating gynaecologic diseases at CT and MRI. RadioGraphics 16:1085-1099. doi:10.1148/radiographics.16.5.8888392

Franks S (2006) Controversy in clinical endocrinology: diagnosis of polycystic ovarian syndrome: in defense of the Rotterdam criteria. J Clin Endocrinol Metab 91:786-789. doi:10.1210/jc.2005-2501

Frisch LS, Copeland KC, Boepple PA (1992) Recurrent ovarian cysts in childhood: diagnosis of McCuneAlbright syndrome by bone scan. Pediatrics 90: 102-104

Garcia-Villanueva M, Badia Figuerola N, Ruiz del Arbol L, Hernandez Ortiz MJ (1990) Zollinger Ellison syndrome due to a borderline mucinous cystadenoma of the ovary. Obstet Gynecol 75:549-552

Ghattamaneni S, Bhuskute N, Weston MJ, Spencer JA (2009) Discriminative MR imaging features of Fallopian tube masses. Clin Radiol 64:815-831. doi:10.1016/j.crad.2009.03.007

Hassen K, Ghossain MA, Rousset P et al (2011) Characterization of papillary projections in benign versus borderline and malignant ovarian masses on conventional and color Doppler ultrasound. AJR Am J Roentgenol 196:1444-1449. doi:10.2214/AJR.10.5014

Hermans RH, Fisher DC, van der Putte HW et al (2003) Adnexal masses in pregnancy. Onkologie 26:167-172. doi: 69838

Heifetz SA, Cushing B, Giller R et al (1998) Immature teratomas in children: pathologic considerations: a report from the combined Pediatric Oncology Group/ Children's Cancer Group. Am J Surg Pathol 22:1115-1124

Hochberg L, Hoffman MS (2017) Differential diagnosis of adnexal mass. www uptodate 2017

Honoré LH, O'Hara KE (1980) Serous papillary neoplasms arising in paramesonephric paraovarian cysts: a report of 8 cases. Acta Obstet Gynecol Scand 59:525-528

Horta M, Cunha TM (2015) Sex cord-stromal tumors of the ovary: a comprehensive review and update for radiologists. Diagn Interv Radiol 21:277-286. doi:10.5152/dir.2015.34414 
Hricak H, Chen M, Coakley FV et al (2000) Complex adnexal masses: detection and characterization with MRI-multivariate analysis. Radiology 214:39-46. doi:10.1148/radiology.214.1.r00ja3939

Johnstone EB, Rosen MP, Neri R et al (2010) The polycystic ovary post-Rotterdam: a common, agedependent finding in ovulatory women without metabolic significance. J Clin Endocrinol Metab 95:4965-4972. doi:10.1210/jc.2010-0202

Jung SE, Lee JM, Rha SE, Byun JY, Jung JI, Hahn ST (2002) CT and MRI of ovarian tumors with emphasis on the differential diagnosis. RadioGraphics 22:13051325. doi:10.1148/rg.226025033

Jung DC, Kim SH, Kim SH (2006) MR imaging findings of ovarian cystadenofibroma and cystadenocarcinofibroma: clues for the differential diagnosis. Korean J Radiol 7:199-204. doi:10.3348/kjr.2006.7.3.199

Kaijser J, Vandecaveye V, Deroose CM et al (2014) Imaging techniques for the pre-surgical diagnosis of adnexal tumours. Best Pract Res Clin Obstet Gynaecol 28:683-695. doi:10.1016/j.bpobgyn.2014.03.013

Kier R (1992) Nonovarian gynaecologic cysts: MR imaging findings. AJR Am J Roentgenol 158:1265-1269. doi:10.2214/ajr.158.6.1590120

Kim JS, Lee HJ, Woo SK, Lee TS (1997) Peritoneal inclusion cysts and their relationship to the ovaries: evaluation with sonography. Radiology 204:481-484. doi:10.1148/radiology.204.2.9240539

Kim JC, Kim SS, Park JY (2000) Bridging vascular sign in the MR diagnosis of exophytic uterine leiomyoma. J Comput Assist Tomogr 24:57-60

Koonings PP, Campbell K, Mishell DR Jr, Grimes DA (1989) Relative frequency of primary ovarian neoplasm: a 10-year review. Obstet Gynecol 74:921-926

Kurman RJ, Ellenson LH, Ronnett B (2011) Blaustein's pathology of the female genital tract, 6th edn. Springer, New York

Kurman RJ, Carcangiu ML, Herrington CS, Young RH (2014) Classification of tumors of the ovary. In: WHO classification of tumours, vol. 6, 4th edn. IARC, Lyon, pp 44-56

Lakhani K, Seifalian AM, Atiomo WU, Hardiman P (2002) Polycystic ovaries. Br J Radiol 75:9-16. doi: $10.1259 /$ bjr.75.889.750009

Laufer MR (2017) Ovarian cysts and neoplasm in infants, children, and adolescents. www.uptodate.com

Lee JH, Jeong YK, Park JK, Hwang JC (2003) “Ovarian vascular pedicle" sign revealing organ of origin of a pelvic mass lesion on helical CT. AJR Am J Roentgenol 181:131-137

Lee TT, Rausch ME (2012) Polycystic ovarian syndrome: role of imaging in diagnosis. RadioGraphics 32:1643-1657

Lengyel E (2010) Ovarian cancer development and metastasis. Am J Pathol 177:1053-1064. doi:10.2353/ ajpath.2010.100105

Levine CD, Patel UJ, Ghanekar D, Wachsberg RH, Simmons MZ, Stein M (1997) Benign extraovarian mimics of ovarian cancer: distinction with imaging studies. Clin Imaging 21:350-358

Levine D, Brown DL, Andreotti RF et al (2010) Management of asymptomatic ovarian and other adnexal cysts imaged at US: society of radiologists in ultrasound consensus conference statement. Radiology 256:943-954. doi:10.1148/radiol.10100213

Lujan ME, Jarrett BY, Brooks ED et al (2013) Updated ultrasound criteria for polycystic ovary syndrome: reliable thresholds for elevated follicle population and ovarian volume. Hum Reprod 28:1361-1368. doi:10.1093/humrep/det062

Matsubayashi R, Matsuo Y, Doi J, Kudo S, Matsuguchi K, Sugimori H (1999) Sclerosing stromal tumor of the ovary: radiologic findings. Eur Radiol 9:1335-1338. doi:10.1007/s003300050844

Matsuki M, Kaji Y, Matsuo M, Kobashi Y (2000) Struma ovarii: MRI findings. $\mathrm{Br} J$ Radiol 73:87-90. doi:10.1259/bjr.73.865.10721328

Moon WJ, Koh BH, Kim SK et al (2000) Brenner tumor of the ovary: CT and MR findings. J Comput Assist Tomogr 24:72-76

Moyle PL, Kataoka MY, Nakai A, Takahata A, Reinhold C, Sala E (2010) Nonovarian cystic lesions of the pelvis. RadioGraphics 30:921-938. doi:10.1148/rg.304095706

Norris HJ, Jensen RD (1972) Relative frequency of ovarian neoplasm in children and adolescents. Cancer 30:713-719

Okada S, Ohaki Y, Inoue K et al (2005) Calcifications in mucinous and serous cystic ovarian tumors. J Nippon Med Sch 72:29-33

Outwater EK, Mitchell DG (1996) Normal ovaries and functional cysts: MR appearance. Radiology 198:397402. doi:10.1148/radiology.198.2.8596839

Palmeiro MM, Cunha TM, Loureiro AL, Esteves G (2016) A rare benign ovarian tumour. BMJ Case Rep (2016) Mar 1;2016. pii: bcr2015214101. doi: 10.1136/ bcr-2015-214101

Patel MD, Ascher SM, Paspulati RM et al (2013) Managing incidental findings on abdominal and pelvic CT and MRI, part 1: white paper of the ACR Incidental Findings Committee II on adnexal findings. J Am Coll Radiol 10:675-681. doi:10.1016/j.jacr.2013.05.023

Rha SE, Byun JY, Jung SE et al (2004) Atypical CT and MRI manifestations of mature ovarian cystic teratomas. AJR Am J Roentgenol 183:743-750. doi:10.2214/ajr.183.3.1830743

Sala EJ, Atri M (2003) MRI of benign adnexal disease. Top Magn Reson Imaging 14:305-327

Saksouk FA, Johnson SC (2004) Recognition of the ovarian origin of pelvic masses with CT. RadioGraphics 24(Suppl 1):S133-S146. doi:10.1148/rg.24si045507

Seidman JD, Russell P, Kurman RJ (2002) Surface epithelial tumors of the ovary. In: Kurman RJ (ed) Blaustein's pathology of the female genital tract. Springer Verlag, New York, pp 791-904 
Sherard GB 3rd, Hodson CA, Williams HJ, Semer DA, Hadi HA, Tait DL (2003) Adnexal masses and pregnancy: a 12-year experience. Am J Obstet Gynecol 189:358-362. discussion 362-363

Spencer JA, Gore RM (2011) The adnexal incidentaloma: a practical approach to management. Cancer Imaging 11:48-51. doi:10.1102/1470-7330.2011.0008

Tanaka YO, Tsunoda H, Kitagawa Y, Ueno T, Yoshikawa H, Saida Y (2004) Functioning ovarian tumors: direct and indirect findings at MR imaging. RadioGraphics 24(Suppl 1):S147-S166. doi:10.1148/rg.24si045501

Tannus JF, Hertzberg BS, Haystead CM, Paulson EK (2009) Unilateral luteoma of pregnancy mimicking a malignant ovarian mass on magnetic resonance and ultrasound. J Magn Reson Imaging 29:713-717. doi:10.1002/jmri.21530

Telischak NA, Yeh BM, Joe BN, Westphalen AC, Poder L, Coakley FV (2008) MRI of adnexal masses in pregnancy. AJR Am J Roentgenol 191:364-370. doi:10.2214/AJR.07.3509

Timmerman D, Moerman P, Vergote I (1995) Meigs syndrome with elevated CA-125 levels: two case reports and review of the literature. Gynecol Oncol 59:405408. doi:10.1006/gyno.1995.9952

Timmerman D, Van Calster B, Testa A et al (2016) Predicting the risk of malignancy in adnexal masses based on the Simple Rules from the International
Ovarian Tumor Analysis group. Am J Obstet Gynecol 214:424-437. doi:10.1016/j.ajog.2016.01.007

Thomassin-Naggara I, Daraï E, Cuenod CA et al (2009) Contribution of diffusion-weighted MR imaging for predicting benignity of complex adnexal masses. Eur Radiol 19:1544-1552. doi:10.1007/s00330-009-1299-4

Thomassin-Naggara I, Toussaint I, Perrot N et al (2011) Characterization of complex adnexal masses: value of adding perfusion- and diffusion-weighted MR imaging to conventional MR imaging. Radiology 258:793803. doi:10.1148/radiol.10100751

van Winter JT, Simmons PS, Podratz KC (1994) Surgically treated adnexal masses in infancy, childhood, and adolescence. Am J Obstet Gynecol 170:1780-1786. discussion 1786-1789

Young RH, Scully RE (2002) Sex cord stromal, steroid cell, and other ovarian tumors. In: Kurman RJ (ed) Blaustein's pathology of the female genital tract. Springer Verlag, New York, pp 903-966

Yamashita Y, Hatanaka Y, Torashima M, Takahashi M, Miyazaki K, Okamura H (1994) Mature cystic teratomas of the ovary without fat in the cystic cavity: MR features in 12 cases. AJR Am J Roentgenol 163:613616. doi:10.2214/ajr.163.3.8079854

Yoshitake T, Asayama Y, Yoshimitsu K et al (2005) Bilateral ovarian leiomyomas: CT and MRI features. Abdom Imaging 30:117-119. doi:10.1007/s00261-004-0202-7 